EDITORIAL NOTE: This paper was published online on 22 January 2019 but was erroneously omitted from the January print issue. The article is presented below for benefit of the print readership. This article should be cited as Joseph W. Baron and Tobias Galla, Phys. Rev. E 99, 012212 (2019).

\title{
Effective diffusion coefficients in reaction-diffusion systems with anomalous transport
}

\author{
Joseph W. Baron $~^{*}$ * and Tobias Galla ${ }^{\dagger}$ \\ Theoretical Physics, School of Physics and Astronomy, The University of Manchester, Manchester M13 9PL, United Kingdom
}

(Received 9 November 2018; published 22 January 2019; corrected 11 March 2019 and 24 September 2019)

\begin{abstract}
We show that the Turing patterns in reaction systems with subdiffusion can be replicated in an effective system with Markovian cross-diffusion. The effective system has the same Turing instability as the original system and the same patterns. If particles are short lived, then the transient dynamics are captured as well. We use the cross-diffusive system to define effective diffusion coefficients for the system with anomalous transport, and we show how they can be used to efficiently describe the Turing instability. We also demonstrate that the mean-squared displacement of a suitably defined ensemble of subdiffusing particles grows linearly with time, with a diffusion coefficient which agrees with our earlier calculations. We verify these deductions by numerically integrating both the fractional reaction-diffusion equation and its normally diffusing counterpart. Our findings suggest that cross-diffusive behavior can come about as a result of anomalous transport.
\end{abstract}

DOI: 10.1103/PhysRevE.99.012212

\section{INTRODUCTION}

In his seminal paper [1] Turing provided a simple mechanism for spontaneous pattern formation in chemical reaction systems. Since this work, the reaction-diffusion paradigm [2] has been used ubiquitously as a tool for the description of pattern-forming phenomena, ranging from predator-prey interactions [3] to developmental biology [4,5].

In this context, one typically assumes that constituents diffuse in a manner such that the mean-squared displacement of individuals grows linearly with time. Mathematically, $\left\langle\mathbf{r}^{2}\right\rangle \sim$ $t$, where $\mathbf{r}$ denotes the position vector of a typical particle and the angular brackets define an average over trajectories. The rate of diffusion can then be quantified by means of the diffusion constant $D=\left\langle\mathbf{r}^{2}\right\rangle /(2 t)[6,7]$. In one spatial dimension this simplifies to $D=\left\langle x^{2}\right\rangle /(2 t)$. However, the situation may not always be this simple. Due to the many complicated interactions between the reactants and their environment, the diffusion may be of an "anomalous" nature. For example, the particles or individuals involved may be subject to "trapping" effects, which can result in subdiffusive behavior: $\left\langle x^{2}\right\rangle \sim t^{\alpha}$, where $0<\alpha<1$ [8-10]. Reaction-diffusion equations with anomalous (or "non-Fickian") diffusion have been used to describe systems ranging from the neolithic transition in Europe [11,12] to photoluminescence in semiconductors [13] to morphogen gradient formation $[14,15]$.

How, then, might one go about defining a quantity which describes the speed of anomalous diffusion? What is the analog of the diffusion constant $D$ ? In order to be useful, such a quantity must not only inform us as to how quickly different reactant types diffuse, but it must also be of physical relevance

\footnotetext{
*joseph.baron@postgrad.manchester.ac.uk

†tobias.galla@manchester.ac.uk
}

Published by the American Physical Society under the terms of the Creative Commons Attribution 4.0 International license. Further distribution of this work must maintain attribution to the author(s) and the published article's title, journal citation, and DOI. and have predictive power. For example, a criterion for pattern formation in normally diffusive reaction-diffusion systems can be written in terms of the diffusion constants and the other system parameters [16]. So, by introducing an "effective diffusion coefficient," we might aim to produce a similar criterion for pattern formation in systems with anomalous diffusion.

The notion of an effective diffusion coefficient has been utilized previously in the study of transport in confined geometries [17] and for tilted periodic potentials [18,19]. The possibility of defining an effective diffusion constant in a reaction-subdiffusion system was mentioned in Ref. [20]. In this paper we present a more detailed argument for the definition of effective diffusion coefficients and seek to interpret the results.

We approach the problem as follows. We start from a reaction-diffusion system in which the reactants can exhibit subdiffusive behavior. We refer to this as the "original" system. We then derive an "effective" normally diffusing reaction-diffusion system, which exhibits pattern formation for the same sets of model parameters as the original anomalously diffusing system. Furthermore, we argue that the normally diffusing system gives rise to the same patterns as the original system in the long-term and, under certain conditions, replicates the transient dynamics of the original system to a good approximation. It is the diffusion coefficients in this normally diffusing system which we use to characterize the pattern formation in the original subdiffusive system. Although we use subdiffusion as the primary example in this work, our method could be applied to other varieties of nonFickian diffusion than just the subdiffusive kind.

We find that the effective diffusion in reaction-diffusion systems with anomalous transport is in general crossdiffusive. That is, the diffusion of one substance may be affected by a concentration gradient in another. As such, we suggest that anomalous transport can be a possible mechanism for the production of cross-diffusive effects in experiments in addition to those that are already known.

We also show that the collective behavior of constituents in the stationary state can give rise to a mean-squared 
displacement which scales linearly with time, $\left\langle x^{2}\right\rangle \sim t$, despite the underlying subdiffusive motion of the individuals. To demonstrate this, we consider an ensemble in which particles are subject to diffusion and removal events but are replaced on removal, thus keeping the ensemble size the same, as would be the case in the homogeneous steady state. The effective diffusion coefficient found from this reasoning agrees with our earlier definitions.

The remainder of this paper is set out as follows: In Sec. II, we present the necessary background information. In particular, we summarize the main features of reactionsubdiffusion systems, which we use in the rest of the work. In Sec. III, we construct the corresponding "effective" normally diffusing system. We go on to show that the condition for Turing pattern formation in the subdiffusive system can be written in terms of the effective diffusion coefficients and that the normally diffusive system produces the same patterns as the original system. Additionally, we show that, under certain circumstances, the normally diffusing system approximates the time-dependent behavior of the original system. The interpretation of the effective diffusion coefficients in terms of the underlying microscopic processes is also discussed. In Sec. IV, we confirm the theoretical results by numerical integration of the reaction-subdiffusion equation and of its normally diffusing counterpart. Finally, we summarize and discuss our findings in Sec. V.

\section{MODEL DEFINITION AND BACKGROUND}

In this section, we discuss the formulation of reactiondiffusion systems in terms of a continuous-time random walk (CTRW) model. The CTRW approach is a standard method by which to derive reaction-diffusion equations with anomalous diffusive behavior [8-10]. We also discuss the stability of the homogeneous steady states in such systems.

\section{A. General reaction-diffusion model}

We consider a system in one spatial dimension which involves different types of particles. These particles may hop from location to location and be involved in reactions. When a reaction occurs, particles of each flavour can be created or destroyed. In principle this defines a stochastic system if the overall particle number is finite. In this paper, however, we ignore the stochastic fluctuations and examine only the deterministic behavior. In doing so, we are assuming that the total number of particles is sufficiently large, so that the fluctuations are comparatively negligible. A further convenient consequence of dealing with large particle numbers is that the discrete quantity of particles can be approximated as a continuous concentration.

Within this approximation, the local particle number density (or concentration) of a particular species $i$ at a time $t$ and at location $x$ is denoted by $\rho_{i}(x, t)$. This means that $\int_{x_{1}}^{x_{2}} \rho_{i}(x, t) d x$ is the number of particles of type $i$ in the region $x_{1}<x<x_{2}$. We write $\rho$ for the vector $\left(\rho_{1}, \rho_{2}, \ldots\right)$, i.e., $\rho(x, t)$ indicates the concentrations of all particles types at a given location and time. Given the initial location of a particle of type $i$, a waiting time is drawn from a distribution $\psi_{i}(t)$. Waiting times for each particle are drawn independently. We do not specify these distributions at this point for the purposes of generality. Each particle then remains where it is until it has waited the allotted time, at which point it hops by a distance $x$, drawn from a hopping kernel $\phi_{i}(x)$. The spatial coordinate $x$ can be continuous or can denote a position on a discrete lattice. Once the particle has hopped, a new waiting time is drawn and the process repeats itself.

Given these definitions, one can define the hazard rate of hopping $h_{i}(t)=\psi_{i}(t) / \Psi_{i}(t)$, where $\Psi_{i}(t)=\int_{t}^{\infty} \psi_{i}(\tau) d \tau$ is the probability that a particle has not hopped for a time $t$ since its last hop. The hazard rate characterizes the proclivity of particles to hop a time $t$ after their last hop. It is constant in the case of regular diffusion but time dependent in the general case.

Particles may also be involved in reactions during their sojourn periods at a fixed location. We label these different types of reactions with an index $r$. They occur with rates which depend on the concentration of particles at that location. The result of a reaction is the local production and/or annihilation of particles. We denote the rate at which a reaction of type $r$ occurs by $\lambda_{r}[\rho(x, t)]$ and the number of particles of type $i$ which are produced or annihilated in such a reaction by $v_{i}^{r}$. If particles are annihilated, then scheduled hopping events involving these particles no longer occur. Particles which are involved in reactions have their waiting times redrawn; this approach applies to irreversible reactions, but special consideration has to be given in the case where the reactions are reversible [21]. We do not consider such reaction schemes in this paper.

In order to deal with the nonconstant hazard rates $h_{i}(t)$ and to derive an equation which describes the time evolution of the concentrations $\rho(x, t)$, one can introduce an additional temporal coordinate $\tau$, the time since the last hop for an individual particle. The concentrations can then be subdivided by introducing the quantities $\rho(x, \tau, t)$ where $\rho(x, t)=$ $\int_{0}^{t} \rho(x, \tau, t) d \tau$. Using such a coordinate, the problem is recast as Markovian. One is then able to derive a generalized reaction-diffusion equation. A detailed derivation of equations of this type can be found in Refs. [11,22]. Carrying out a calculation along these lines, one obtains

$$
\begin{aligned}
\frac{\partial \rho_{i}(x, t)}{\partial t}= & \int d x^{\prime}\left[\phi_{i}\left(x-x^{\prime}\right)-\delta\left(x-x^{\prime}\right)\right] \int_{0}^{t} d \tau K_{i}(\tau) \\
& \times \rho_{i}\left(x^{\prime}, t-\tau\right) e^{-\int_{t-\tau}^{t} d t^{\prime} \frac{R_{i}^{-}\left[\rho\left(x^{\prime}, t^{\prime}\right)\right]}{\rho_{i}\left(x^{\prime}, t^{\prime}\right)}} \\
& +R_{i}^{+}[\rho(x, t)]-R_{i}^{-}[\rho(x, t)]
\end{aligned}
$$

We have defined the following objects:

$$
\begin{aligned}
& R_{i}^{+}[\rho(x, t)]=\sum_{r} H\left(v_{i}^{r}\right) \lambda_{r}[\rho(x, t)] v_{i}^{r}, \\
& R_{i}^{-}[\rho(x, t)]=\sum_{r} H\left(-v_{i}^{r}\right) \lambda_{r}[\rho(x, t)] v_{i}^{r},
\end{aligned}
$$

where $H(u)=1$ if $u \geqslant 0$ and $H(u)=0$ otherwise. The quantities $R_{i}^{ \pm}$are the net production and annihilation rates, respectively, of particles of type $i$. In Eq. (1) $K_{i}(\tau)$ is the "memory kernel" defined through its Laplace transform,

$$
\mathcal{L}_{t}\left\{K_{i}(t)\right\}=\frac{\mathcal{L}_{t}\{\psi(t)\}}{\mathcal{L}_{t}\{\Psi(t)\}} .
$$


The Laplace transform is given by $\mathcal{L}_{t}\{f(t)\}(u)=$ $\int_{0}^{\infty} e^{-u t} f(t) d t$.

In Eq. (1), the reaction term, $R^{+}(\rho)-R^{-}(\rho)$, and the diffusion term [which contains convolutions with both the hopping kernel $\phi(x)$ and the memory kernel $\left.K_{i}(t)\right]$ are coupled via the exponential factor in the convolution with the memory kernel. This coupling arises from the fact that particles may be annihilated before they hop: If the death rate $R_{i}^{-}$were identically zero, then the contribution of the concentration at a time $t-\tau$ to the integral over time in Eq. (1) would be $K_{i}(\tau) \rho_{i}\left(x^{\prime}, t-\tau\right)$. To account for a nonzero death rate, this contribution is weighted by the probability that a particle of type $i$ survives the time interval from $t-\tau$ to $t$; this probability is given by $e^{-\int_{t-\tau}^{t} d t^{\prime} \frac{R_{i}^{-}\left[\rho\left(x^{\prime}, t^{\prime}\right)\right]}{\rho_{i}\left(x^{\prime}, t^{\prime}\right)}}$. This coupling term is absent in the case of Markovian hopping. That is, if we choose the exponential waiting time distribution $\psi_{i}(t)=\frac{1}{\tau_{i}} e^{-\frac{t}{\tau_{i}}}$, then we obtain $K_{i}(t)=\frac{1}{\tau_{i}} \delta(t)$, and the reaction and diffusion terms in Eq. (1) decouple. In general though, the time evolution of the concentrations depends on the history of the system, and the process is manifestly non-Markovian.

\section{B. Reaction-subdiffusion system}

In this paper, we will primarily concern ourselves with a specific case of non-Markovian transport: subdiffusion. We choose the waiting-time distribution to be the Mittag-Leffler distribution [23]

$$
\psi_{i}(t)=-\frac{d}{d t} E_{\alpha_{i}}\left[-\left(t / \tau_{i}\right)^{\alpha_{i}}\right]
$$

where $0<\alpha_{i}<1$ and where $E_{\alpha_{i}}(\cdot)$ is the one-parameter Mittag-Leffler function [24]. This function can be thought of as a generalized exponential function and is defined as

$$
E_{\alpha_{i}}(x)=\sum_{k=0}^{\infty} \frac{x^{k}}{\Gamma\left(\alpha_{i} k+1\right)},
$$

where $\Gamma(\cdot)$ denotes the standard gamma function. The constant $\tau_{i}$ has units of time; its role is to fix the overall timescale of the hopping process. It is important to note that the Mittag-Leffler distribution has the "heavy-tail" property $\lim _{T \rightarrow \infty} \int_{0}^{T} t \psi_{i}(t) d t \rightarrow \infty$. That is, in absence of annihilation the first moment of the waiting times between hops diverges. This makes the Mittag-Leffler function suitable for modeling the hopping of particles subject to "trapping" effects [8].

We allow the hopping kernel to take a general symmetrical form. If one presumes that the hopping kernel is of the form $\phi_{i}(x)=\Phi_{i}(x / \xi)$, where $\xi$ characterizes the typical hopping distance and $\Phi_{i}(\cdot)$ is even, then the Fourier transform of the hopping kernel has following asymptotics:

$$
\tilde{\phi}_{i}(k) \approx 1-\sigma_{i}^{2} \xi^{2} k^{2}+O\left(\xi^{4}\right)
$$

for small $\xi$, where $\sigma_{i}$ is a constant related to the variance of the hopping distances such that $-\left.\frac{\partial^{2}}{\partial k^{2}} \tilde{\phi}(k)\right|_{k=0}=2 \sigma_{i}^{2} \xi^{2}$.

If one were to track the movement of an ensemble of particles which hopped with waiting-times drawn from the distribution in Eq. (4) and hopping distances drawn from a hopping kernel described by Eq. (6), one would obtain subdiffusive behavior, characterized by the mean-squared displacement $\left\langle x^{2}\right\rangle \sim t^{\alpha_{i}}$ [8]. This can be shown using the Montroll-Weiss formula [25]. The parameter $\alpha_{i}$ characterizes the degree to which the diffusion is subdiffusive, with $\alpha_{i} \rightarrow 0$ describing extremely subdiffusive behavior and $\alpha_{i}=1$ leading to normal diffusion. We refer to $\alpha_{i}$ as the anomalous exponent.

The Laplace transform of the memory kernel [see Eq. (3)] for the distribution in Eq. (4) has the following simple form [26]:

$$
\mathcal{L}_{t}\left\{K_{i}(t)\right\}(u)=\frac{u^{1-\alpha_{i}}}{\tau_{i}^{\alpha_{i}}} .
$$

However, the Laplace transform for this function cannot easily be inverted. This makes it difficult to find the memory kernel $K_{i}(t)$ in the time domain in explicit form. As a result, it is not convenient to write the reaction-diffusion equation in the form of Eq. (1). Instead, we use the Riemann-Liouville fractional derivative, defined as [27]

$$
{ }_{0} D_{t}^{1-\alpha} f(t)=\frac{1}{\Gamma(\alpha)} \frac{\partial}{\partial t} \int_{0}^{t} \frac{f\left(t^{\prime}\right)}{\left(t-t^{\prime}\right)^{1-\alpha}} d t^{\prime} .
$$

This has the following property:

$$
\mathcal{L}_{t}\left\{{ }_{0} D_{t}^{1-\alpha} f(t)\right\}(u)=u^{1-\alpha} \mathcal{L}_{t}\{f\}(u),
$$

provided that $\lim _{t \rightarrow 0} \int_{0}^{t} f(\tau)(t-\tau)^{\alpha-1} d \tau=0$, which is satisfied if $f(t)$ is continuous and $f^{\prime}(t)$ is integrable [27]. It is reasonable to assume that this condition applies to the functions on which the fractional derivative acts in this work, as these functions are related to particle concentrations.

Using these definitions, we define the rescaled time $\eta_{i}=$ $\tau_{i} \xi^{-2 / \alpha_{i}}$. This rescaling hints at the underlying subdiffusive nature of the transport. Taking the limit $\xi \rightarrow 0$, Eq. (1) can be written as

$$
\frac{\partial \rho_{i}(x, t)}{\partial t}=\frac{\sigma_{i}^{2}}{\eta_{i}^{\alpha_{i}}} \frac{\partial^{2}}{\partial x^{2}} M_{i}(x, t)+f_{i}(x, t),
$$

where $f_{i} \equiv R_{i}^{+}[\rho(x, t)]-R_{i}^{-}[\rho(x, t)]$ is the total reaction rate and where we define

$$
\begin{aligned}
M_{i}(x, t)= & e^{-\int_{0}^{t} \frac{R_{i}^{-}\left[\rho\left(x, t^{\prime}\right)\right]}{\rho_{i}\left(x, t^{\prime}\right)} d t^{\prime}} \\
& \times{ }_{0} D_{t}^{1-\alpha_{i}}\left\{e^{\int_{0}^{t} \frac{R_{i}^{-}\left[\rho\left(x, t^{\prime}\right)\right]}{\rho_{i}\left(x, t^{\prime}\right)} d t^{\prime}} \rho_{i}(x, t)\right\} .
\end{aligned}
$$

The history-dependent quantity $M_{i}(x, t)$ characterizes the current number of particles of type $i$ hopping away from location $x$ per unit time. References $[11,14,22]$ contain a more detailed discussion of the origins of Eq. (10). In a similar way to Eq. (1), the reaction and diffusion terms in Eq. (10) are coupled through exponential terms in the fractional derivative. We note that the evolution of the concentrations described by Eq. (10) is dependent on the history of the system and is therefore non-Markovian. However, for $\alpha_{i}=1$ the Mittag-Leffler distribution Eq. (4) reduces to an exponential distribution, and one obtains the normal reaction-diffusion equation,

$$
\frac{\partial \rho_{i}(x, t)}{\partial t}=\frac{\sigma_{i}^{2}}{\eta_{i}} \frac{\partial^{2}}{\partial x^{2}} \rho_{i}(x, t)+f_{i}(x, t) .
$$

In this case the length of time since the last hop is irrelevant to the current hopping rate, and the process is Markovian. 
We note that Eq. (10) can also be used as an approximation to the dynamics when a general class of heavy-tailed waitingtime distributions is used, not just the Mittag-Leffler function $[8,9,14]$.

\section{Linear stability and pattern formation in the reaction-subdiffusion system}

Now that we have discussed the general formulation of reaction-subdiffusion systems, we consider the stability of their fixed points and discuss conditions for pattern formation. From here on in, we will focus on systems which have two species of reactants.

A stable fixed point in a nonspatial system can become unstable with the introduction of diffusive proceses. As a result of the instability, a limited band of Fourier modes with nonzero wave numbers can become excited, resulting in the formation of stationary patterns [2,16]. Turing [1] demonstrated this effect in reaction-diffusion systems with normal diffusion, such as the one described by Eq. (12) and established a condition for such an instability to occur.

A similar instability has been found in the reactionsubdiffusion system whose evolution is described by Eq. (10), see Refs. [20,22]. We denote the homogeneous fixed point of this system by $\rho^{0}$, which is defined by $f_{i}\left(\rho^{0}\right)=0$ for all $i$. Writing the derivatives of $f_{i}$ with respect to particle concentrations as $f_{i j}=\frac{\partial f_{i}\left(\rho^{0}\right)}{\partial \rho_{j}^{0}}$, the stability of this fixed point against uniform perturbations requires $[2,16]$

$$
f_{11}+f_{22}<0, \quad f_{11} f_{22}-f_{12} f_{21}>0 .
$$

Unless indicated otherwise the $f_{i j}$ are always to be evaluated at the homogeneous fixed point.

We denote deviations from the homogeneous fixed point by $\delta \rho_{i}(x, t) \equiv \rho_{i}(x, t)-\rho_{i}^{0}$. Writing $\delta \tilde{\rho}_{i}(k, t)$ for the Fourier transform with respect to the spatial coordinate, one finds the following dynamics for the Fourier mode with wave number $k$,

$$
\begin{aligned}
\frac{\partial \delta \tilde{\rho}_{i}(k, t)}{\partial t}= & -k^{2} \frac{\sigma_{i}^{2}}{\eta_{i}^{\alpha_{i}}} e^{-p_{i} t}\left[\sum_{j} A_{i j} \rho_{i}^{0} D_{t}^{1-\alpha_{i}}\left\{e^{p_{i} t} \int_{0}^{t} \delta \tilde{\rho}_{j}\left(k, t^{\prime}\right) d t^{\prime}\right\}-\sum_{j} A_{i j} \int_{0}^{t} \delta \tilde{\rho}_{j}\left(k, t^{\prime}\right) d t^{\prime}{ }_{0} D_{t}^{1-\alpha_{i}}\left\{e^{p_{i} t} \rho_{i}^{0}\right\}\right. \\
& \left.+{ }_{0} D_{t}^{1-\alpha_{i}}\left\{e^{p_{i} t} \delta \tilde{\rho}_{i}(k, t)\right\}\right]+\sum_{j} f_{i j} \delta \tilde{\rho}_{j}(k, t),
\end{aligned}
$$

to linear order in the perturbations. We have written $p_{i}=R_{i}^{-}\left(\rho^{0}\right) / \rho_{i}^{0}$ for the per capita removal rate of species $i$ at the fixed point. We have also introduced $A_{i j}=\frac{\partial p_{i}\left(\rho^{0}\right)}{\partial \rho_{j}^{0}}$. To keep the notation compact we have omitted the argument $\rho^{0}$ in Eq. (14); it is to be understood that the $A_{i j}$ are evaluated at the homogeneous fixed point.

In Ref. [22] a condition for the Fourier mode $k$ to be unstable to perturbations was derived for a system with one subdiffusing species and one normally diffusing species. A generalized version of this result for the case where both species subdiffuse is

$$
\begin{gathered}
\left\{f_{11}-k^{2} \frac{\sigma_{1}^{2}}{\eta_{1}^{\alpha_{1}}}\left[p_{1}^{1-\alpha_{1}}+A_{11} \rho_{1}^{0} p_{1}^{-\alpha_{1}}\left(1-\alpha_{1}\right)\right]\right\}\left\{f_{22}-k^{2} \frac{\sigma_{2}^{2}}{\eta_{2}^{\alpha_{2}}}\left[p_{2}^{1-\alpha_{2}}+A_{22} \rho_{2}^{0} p_{2}^{-\alpha_{2}}\left(1-\alpha_{2}\right)\right]\right\} \\
-\left\{f_{12}-k^{2} \frac{\sigma_{1}^{2}}{\eta_{1}^{\alpha_{1}}}\left[A_{12} \rho_{1}^{0} p_{1}^{-\alpha_{1}}\left(1-\alpha_{1}\right)\right]\right\}\left\{f_{21}-k^{2} \frac{\sigma_{2}^{2}}{\eta_{2}^{\alpha_{2}}}\left[A_{21} \rho_{2}^{0} p_{2}^{-\alpha_{2}}\left(1-\alpha_{2}\right)\right]\right\}<0 .
\end{gathered}
$$

A derivation of this formula is detailed in Appendix A 1; we use an alternative route to that of Ref. [22]. We note that a sign error was made in the calculation in Ref. [22]. This mistake has been corrected in Eq. (15), see also Appendix A 2.

\section{CONSTRUCTION OF THE EFFECTIVE NORMALLY DIFFUSIVE SYSTEM}

\section{A. Definition of the system and stationary patterns}

In the following, we construct a Markovian system, with normal diffusion, which replicates the stationary properties of the reaction-subdiffusion system. Finding a Markovian system such as this allows us to define effective diffusion coefficients and to interpret the behavior in the original system more easily. We also discuss the conditions under which the transient dynamics of the original system can be approximated by the time-dependent behavior of this effective Markovian system.

At first it may seem surprising that one should be able to replicate the features of a manifestly non-Markovian system with a Markovian system. However, suppose that, in the longterm, the reaction-subdiffusion system reaches a stationary state. This state may be patterned or homogeneous. Since the non-Markovian "memory" effects in the system are only detectable in dynamic quantities, one might suspect that the stationary behavior can be described without the use of a nonMarkovian memory kernel or a fractional derivative. In this case, one ought to be able to write down a Markovian system which has the same stationary properties as the original nonMarkovian system.

We propose a Markovian system of the form

$$
\begin{aligned}
\frac{\partial \rho_{i}(x, t)}{\partial t} & =\frac{\partial^{2}}{\partial x^{2}}\left[\hat{D}_{i}(\boldsymbol{\rho}) \rho_{i}\right]+f_{i}(\boldsymbol{\rho}) \\
& =\frac{\partial}{\partial x}\left[D_{i 1}(\boldsymbol{\rho}) \frac{\partial \rho_{1}}{\partial x}+D_{i 2}(\boldsymbol{\rho}) \frac{\partial \rho_{2}}{\partial x}\right]+f_{i}(\boldsymbol{\rho}),
\end{aligned}
$$

where we define

$$
D_{i j}(\boldsymbol{\rho}) \equiv \frac{\partial}{\partial \rho_{j}}\left[\hat{D}_{i}(\boldsymbol{\rho}) \rho_{i}(x)\right] .
$$


The specific choice of $\hat{D}_{i}(\boldsymbol{\rho})$, which replicates the stationary properties of the original system, will be described below. The system in Eq. (16) has the same reaction terms as that in Eq. (10), but the anomalous diffusion term has been replaced by a cross-diffusion term. The (effective) diffusion coefficient $\hat{D}_{i}$ depends on the local concentration vector $\rho(x, t)$. That is, the transport of one substance can be affected by the presence or absence of particles of all types. It is important to note that the diffusion term in Eq. (16) is not dependent on the history of the system.

To motivate the proposed effective cross-diffusive dynamics and to define the effective diffusion coefficients $\hat{D}_{i}$, we first focus on the condition for the instability of a perturbation with wave number $k$. In the system described by Eq. (16) this mode is unstable if

$$
\begin{aligned}
& {\left[f_{11}-k^{2} D_{11}\left(\boldsymbol{\rho}^{0}\right)\right]\left[f_{22}-k^{2} D_{22}\left(\boldsymbol{\rho}^{0}\right)\right]} \\
& \quad-\left[f_{12}-k^{2} D_{12}\left(\boldsymbol{\rho}^{0}\right)\right]\left[f_{21}-k^{2} D_{21}\left(\boldsymbol{\rho}^{0}\right)\right]<0 .
\end{aligned}
$$

A detailed derivation of Eq. (18) is given in Refs. [28,29]. We define the effective diffusion coefficients for the subdiffusive system by requiring that Eq. (18) is the same condition as Eq. (15). The following choice satisfies this requirement:

$$
\hat{D}_{i}(\rho)=\frac{\sigma_{i}^{2}}{\eta_{i}^{\alpha_{i}}}\left\{\frac{R_{i}^{-}[\rho(x)]}{\rho_{i}(x)}\right\}^{1-\alpha_{i}} .
$$

Making this choice we obtain

$$
D_{i j}\left(\rho^{0}\right)=\frac{\sigma_{i}^{2}}{\eta_{i}^{\alpha_{i}}}\left[p_{i}^{1-\alpha_{i}} \delta_{i j}+A_{i j} \rho_{i}^{0} p_{i}^{-\alpha_{i}}\left(1-\alpha_{i}\right)\right] .
$$

By choosing the effective diffusion coefficients in this way, we ensure that the Markovian system and the original subdiffusive system experience the Turing instability for the same sets of system parameters. Furthermore, the same sets of modes are unstable in the Markovian system as in the original subdiffusive system.

However, having the same sets of unstable modes does not necessarily mean that the two systems converge to the same patterned states in the long-time limit. This is because in order for the steady-state patterns to be produced, the exponential growth of the unstable modes must be curtailed by the nonlinearities in the reaction rates. This is not accounted for in the linear stability analysis. That being said, one can indeed show that the stationary patterns produced in the both systems obey the same stationary equation. We first note the following property of the Riemann-Liouville derivative:

$$
\begin{aligned}
{ }_{0} D_{t}^{1-\alpha}\left\{e^{p t}\right\} & =\frac{1}{\Gamma(\alpha)} \frac{\partial}{\partial t} \int_{0}^{t} \frac{e^{p(t-\tau)}}{\tau^{1-\alpha}} d \tau \\
& =\frac{1}{\Gamma(\alpha)}\left[t^{\alpha-1}+e^{p t} \int_{0}^{t} p \tau^{\alpha-1} e^{-p \tau} d t^{\prime}\right] \\
& =\frac{1}{\Gamma(\alpha)}\left\{t^{\alpha-1}+e^{p t} p^{1-\alpha}[\Gamma(\alpha)-\Gamma(\alpha, p t)]\right\},
\end{aligned}
$$

where $\Gamma(\alpha, x)=\int_{x}^{\infty} s^{\alpha-1} e^{-s} d s$ is the upper incomplete gamma function [30], which has the property $\lim _{x \rightarrow \infty} \frac{\Gamma(\alpha, x)}{x^{\alpha-1} e^{-x}}=1$. Therefore, for $t \gg \frac{1}{p}$,

Using Eq. (22) in combination with Eq. (10) and the fact that the concentrations do not vary in time in the stationary state, one can deduce that the stationary state in the subdiffusive system obeys

$$
\frac{\sigma_{i}^{2}}{\eta_{i}^{\alpha_{i}}} \frac{\partial^{2}}{\partial x^{2}}\left(\left\{\frac{R_{i}^{-}[\rho(x)]}{\rho_{i}(x)}\right\}^{1-\alpha_{i}} \rho_{i}(x)\right)+f_{i}[\rho(x)]=0,
$$

which, if one notes the definition of $\hat{D}_{i}(\rho)$ in Eq. (19), is the relation defining the stationary state of Eq. (16).

\section{B. Critical ratio of the effective coefficients required for pattern formation}

So far, we have discussed conditions for the instability of perturbations with specific wave numbers $k$. We now proceed to establish a condition for the onset of Turing patterns in the subdiffusive system. Such a criterion, a version of which has been written down before in Ref. [20], is made far simpler with the use of the effective diffusion coefficients in Eq. (17). Since the effective Markovian system has been constructed so that the criterion for the instability of a particular mode $k$ is the same as in the original subdiffusive system, the criterion for Turing pattern formation in general will also be the same in both systems. We therefore work with the effective system.

Turing patterns are formed when a finite range of Fourier modes with nonzero wave numbers is unstable but the $k=0$ mode is stable, such that Eq. (13) is satisfied. In order to find a condition for the formation of Turing patterns, one identifies the value of $k^{2}$ for which the left-hand side of Eq. (18) is minimal. We refer to this value as the "critical" Fourier mode. In order for any other mode to satisfy Eq. (18), this critical mode must also do so. One finds

$$
k_{\text {crit }}^{2}=\frac{D_{21} f_{12}+D_{12} f_{21}-D_{22} f_{11}-D_{11} f_{22}}{2\left(D_{12} D_{21}-D_{11} D_{22}\right)},
$$

where we keep in mind that $D_{i j}=D_{i j}\left(\rho^{0}\right)$. Substituting this result into Eq. (18), one obtains the following condition for the presence of Turing patterns [28,29]:

$$
\begin{aligned}
\frac{D_{22}}{D_{11}}> & \left\{\frac { 1 } { f _ { 1 1 } } \left[\sqrt{f_{11} f_{22}-f_{12} f_{21}}\right.\right. \\
& \left. \pm \sqrt{\left.-\left(f_{12}-\frac{D_{12}}{D_{11}} f_{11}\right)\left(f_{21}-\frac{D_{21}}{D_{11}} f_{11}\right)\right]}\right\}^{2}-\frac{D_{21} D_{12}}{D_{11}^{2}} .
\end{aligned}
$$

When the effective cross-diffusion terms are zero $\left(D_{12}=\right.$ $D_{21}=0$ ) this reduces to the condition for Turing instability in regular reaction-diffusion systems [16],

$$
\frac{D_{22}}{D_{11}}>\left[\frac{1}{f_{1}}\left(\sqrt{f_{11} f_{22}-f_{12} f_{21}} \pm \sqrt{-f_{12} f_{21}}\right)\right]^{2} .
$$

This reduction to a known result for diffusive systems underlines the fact that the effective diffusion coefficients encapsulate the net diffusivity in the reaction-subdiffusion system. 
For the cross-terms to be zero, the death rates of both species must be independent of the concentration of the other or the diffusion of both species must be Markovian. The choice of whether the criteria Eq. (25) and Eq. (26) contain a plus or a minus sign is determined by which of species 1 or 2 is the activator (inhibitor). This can be seen realizing that the critical ratio of the inhibitor diffusion constant to that of the activator for Turing pattern formation to occur should reduce to a number which is greater than unity in the normally diffusing case [5,20]. If species 1 is the activator, then Eq. (25) and Eq. (26) should contain a plus sign.

\section{Limit of large removal rates}

Naturally, the Markovian and subdiffusive systems described by Eqs. (10) and (16) do not, in general, have identical dynamics. However, one can show that the dynamics of the subdiffusive system are replicated to a good approximation in the corresponding effective Markovian system, when the particle removal rates are large. In this limit, particles survive for only short amounts of time on average, so the time horizon for memory effects is also small and, consequently, the dynamics can be approximated as memoryless.

To show this, let $p_{i}(x, t)=\frac{R_{i}^{-}[\rho(x, t)]}{\rho_{i}(x, t)}$ be the per capita removal rate for particles of type $i$. When the death rate is sufficiently large, one obtains the following:

$$
\begin{aligned}
{ }_{0} D_{t}^{1-\alpha_{i}} & \left\{\rho_{i}(x, t) e^{\int_{0}^{t} p_{i}\left(x, t^{\prime}\right) d t^{\prime}}\right\} \\
& \approx \rho_{i}(x, t) p_{i}(x, t)^{1-\alpha_{i}} e^{\int_{0}^{t} p_{i}\left(x, t^{\prime}\right) d t^{\prime}} .
\end{aligned}
$$

We refer the reader to Appendix B for further details of this approximation. Noting that $\frac{\sigma_{i}^{2}}{\eta_{i}{ }_{i}} p_{i}(x, t)^{1-\alpha_{i}}=\hat{D}_{i}[\rho(x, t)]$, the right-hand sides of Eqs. (10) and (16) are found to be approximately equal in the regime in which the approximation Eq. (27) is valid.

\section{Interpretation}

We have shown that an appropriately constructed normally diffusive system can replicate some of the most important features of the original subdiffusive system, in particular the location of any Turing instability in parameter space, and the resulting patterns. This has allowed us to define effective diffusion coefficients for the subdiffusive system. In this section, we provide further insight as to why this is possible.

Before we do this, however, let us first contrast the effective diffusion coefficients $\hat{D}_{i}(\rho)$ with the quantities $D_{i j}(\boldsymbol{\rho})$ [see Eq. (17)]. The coefficients $\hat{D}_{i}(\rho)$ appear inside the secondorder spatial derivative in Eq. (16); they represent the proclivity of particles to hop away from a given location. The coefficient $D_{i j}(\rho)$, on the other hand, describes how the transport of particles of type $i$ is affected by a local gradient of the concentration of particles of type $j$. Which set of coefficients one decides to use is a matter of taste. The coefficients $D_{i j}(\rho)$ have the advantage of highlighting the cross-diffusive nature of the system while the $\hat{D}_{i}(\rho)$ are more succinct. The $D_{i j}(\rho)$ are also useful for writing down the criterion for Turing instability, as was demonstrated in Sec. III B.

So far, we have introduced effective diffusion coefficients for subdiffusive systems in the context of Eq. (16), but we have not related them to the statistics of particle trajectories. We would now like to provide further interpretation and establish how the well-known diffusive law $\left\langle x^{2}\right\rangle=2 D t$ can be obtained in the context of subdiffusive systems. As we will see this can be achieved by defining a suitable ensemble of particles, and the connection with the effective diffusion coefficients can be made.

To do this, we consider the following equation:

$$
\begin{aligned}
\frac{\partial P_{i}(x, t)}{\partial t} & =\frac{\sigma_{i}^{2}}{\eta_{i}^{\alpha_{i}}} \frac{\partial^{2}}{\partial x^{2}}\left\{e^{-p_{i} t} D_{t}^{1-\alpha_{i}}\left[e^{p_{i} t} P_{i}(x, t)\right]\right\} \\
& -\theta p_{i} P_{i}(x, t)
\end{aligned}
$$

in which $\theta$ is a parameter which may take values 0 or 1 . Equation (28) encapsulates the subdiffusive terms of Eq. (10) and, for $\theta=1$, the annihilation reactions when the removal rate $p_{i}$ is constant and uniform (as it would be the case in the homogeneous steady state). In the original system, subdiffusive motion and removal are the only processes an individual particle can undergo once it has been created. We assume that the initial condition at time $t=0$ is given by $P_{i}(x, t=0)=\delta(x)$. Let us discuss possible microscopic processes described by Eq. (28).

For $\theta=1$, the equation describes a system in which particles subdiffuse by drawing waiting times from the MittagLeffler distribution and in which they are also subject to a constant removal rate $p_{i}$. The solution $P_{i}(x, t)$ of Eq. (28) describes the density of particles of type $i$ present at time $t$ at position $x$. The total number of particles in the system, $\int P_{i}(x, t) d x=e^{-p_{i} t}$, decreases with time. We define $C_{i}(x, t) \equiv P_{i}(x, t) /\left[\int P_{i}\left(x^{\prime}, t\right) d x^{\prime}\right]$; this is the probability density for the position of surviving particles, that is, particles still present in the system at time $t$.

The subdiffusive law is obtained by examining the meansquared displacement of these surviving particles. To see this we define $\left\langle x(t)^{2}\right\rangle_{\text {surv }}=\int C_{i}(x, t) x^{2} d x$. One then finds

$$
\left\langle x(t)^{2}\right\rangle_{\mathrm{surv}}=2 \frac{\sigma_{i}^{2}}{\eta_{i}^{\alpha}} \frac{1}{\Gamma\left(1+\alpha_{i}\right)} t^{\alpha_{i}} .
$$

Equation (29) is derived in Appendix C.

We now turn to the the case $\theta=0$, which can be obtained from the scenario for $\theta=1$ by adding a term $p_{i} P_{i}(x, t)$ on the right-hand side of Eq. (28). This indicates additional particle production. More precisely, one representation of the case $\theta=$ 0 is a dynamics in which any particle that is removed is immediately replaced by another identical particle, which draws a new waiting time. This reflects the behavior in the stationary state of the full system in Eq. (1). In the stationary state the particle number at each location is constant, and $R_{i}^{+}\left(\rho^{0}\right)=$ $R_{i}^{-}\left(\rho^{0}\right)$. The per capita removal rate of particles of type $i$ is given by $p_{i}=R_{i}^{-}\left(\rho^{0}\right) / \rho_{i}^{0}$ and removal and production balance. The density $P_{i}(x, t)$ remains normalized at all times for $\theta=0$, and we define $\left\langle x(t)^{2}\right\rangle_{\text {replace }}=\int P_{i}(x, t) x^{2} d x$. It is this ensemble which leads to diffusive behavior,

$$
\left\langle x(t)^{2}\right\rangle_{\text {replace }} \approx 2 \frac{\sigma_{i}^{2}}{\eta_{i}^{\alpha}} p_{i}^{1-\alpha_{i}} t .
$$

This relation is again derived in Appendix C. 
From Eq. (30), one can read off the effective diffusion coefficient $\hat{D}_{i}\left(\boldsymbol{\rho}^{0}\right)=\frac{\sigma_{i}^{2}}{\eta_{i}^{\alpha}} p_{i}^{1-\alpha_{i}}$. This coincides with the earlier definition in Eq. (19).

In summary, if one includes the removal term in Eq. (28) $(\theta=1)$, then one finds that the ensemble of surviving particles generates subdiffusive statistics. If this term is not present (i.e., removed particles are replaced), then standard diffusive statistics $\left\langle x(t)^{2}\right\rangle \propto t$ ensue, despite the nonstandard transport term in Eq. (28).

\section{VERIFICATION USING NUMERICAL INTEGRATION}

\section{A. Lengyel-Epstein model}

To test the conclusions of Sec. III, we numerically integrate the reaction-subdiffusion-equation (10) and the corresponding normally diffusive system in Eq. (16) and then compare the numerical solutions. We do this for the example of the Lengyel-Epstein model [31], which was introduced primarily as a way of modeling the $\mathrm{ClO}_{2}^{-}-\mathrm{I}^{-}-\mathrm{MA}$ reaction. This chemical system exhibits Turing patterns experimentally $[32,33]$. We use a simplified two-species version of the full model and focus on the case of one spatial dimension. The system involves two types of particles, labeled A and B, which each undergo a (sub)diffusion process, potentially with different anomalous exponents. Particles at the same location can also react. The reactions are described by the terms

$$
\begin{aligned}
& f_{A}(\boldsymbol{\rho})=a N-b \rho_{A}-4 \frac{c N \rho_{A} \rho_{B}}{d N^{2}+\rho_{A}^{2}}, \\
& f_{B}(\boldsymbol{\rho})=b \rho_{A}-\frac{c N \rho_{A} \rho_{B}}{d N^{2}+\rho_{A}^{2}},
\end{aligned}
$$

where $\rho_{A}$ and $\rho_{B}$ are the particle concentrations and where $a, b, c, d$, and $N$ are positive model parameters. The first term in the definition of $f_{A}$ describes the production of particles of type $\mathrm{A}$, and the first term in $f_{B}$ represents the production of $\mathrm{B}$ particles. The remaining terms describe removal processes; the corresponding per capita death rates for the two species are given by

$$
\frac{R_{A}^{-}}{\rho_{A}}=b+4 \frac{c N \rho_{B}}{d N^{2}+\rho_{A}^{2}}, \quad \frac{R_{B}^{-}}{\rho_{B}}=\frac{c N \rho_{B}}{d N^{2}+\rho_{A}^{2}} .
$$

The Lengyel-Epstein system has a homogeneous deterministic fixed point at $\rho_{A}^{0}=\frac{a N}{5 b}, \rho_{B}^{0}=\frac{b d N}{c}\left[1+\left(\frac{\rho_{A}^{0}}{\sqrt{d} N}\right)^{2}\right]$. Using Eq. (13), the homogeneous fixed point in the well-mixed system is stable so long as $c a>\frac{3}{5} a^{2}-25 b^{2} d$.

\section{B. Numerical integration method}

The numerical integration of Eq. (10) is made difficult by the fact that the evolution of the system depends not only on the concentrations at the present time but also on the concentrations at all earlier times. This is due to the nature of the fractional derivative ${ }_{0} D_{t}^{1-\alpha}$, defined in Eq. (8). Further complications arise from the coupling of reaction and subdiffusion terms, viz., the presence of the exponential term inside the fractional derivative in Eq. (10). To our knowledge, most existing numerical methods for integrating reaction-subdiffusion equations have not taken this coupling into account [34-36].

The key to integrating Eq. (10) is in recognizing that the fractional derivative can be expressed in discretized form using the equivalence of the Grünwald-Letnikov and the Riemann-Liouville definitions of the fractional derivative, which is valid when $f(t)$ is continuous and $f^{\prime}(t)$ is integrable [27,37],

$$
{ }_{0} D_{t}^{1-\alpha} f(t)=\frac{1}{(\Delta t)^{\alpha}} \sum_{j=0}^{\left[\frac{t}{\Delta t}\right]}(-1)^{j}\left(\begin{array}{c}
\alpha \\
j
\end{array}\right) f(t-j \Delta t)+O(\Delta t)
$$

where $[x]$ denotes the integer part of $x$ and where $\left(\begin{array}{l}\alpha \\ j\end{array}\right)$ is the generalized binomial coefficient

$$
\left(\begin{array}{l}
\alpha \\
j
\end{array}\right)=\frac{\alpha(\alpha-1)(\alpha-2) \cdots(\alpha-j+1)}{j !} .
$$

The numerical approach is then based on first-order Eulerforward integration. We also discretize space and operate on a one-dimensional lattice with spacing $\Delta x$ and periodic boundary conditions. The Laplacian in one dimension, $\frac{\partial^{2} f}{\partial x^{2}}(x)$, is discretized as $[f(x-\Delta x)-2 f(x)+f(x+\Delta x)] / \Delta x^{2}$. At each time step the fractional derivatives ${ }_{0} D_{t}^{1-\alpha}$ $\left[\exp \left\{\int_{0}^{t} \frac{R_{i}^{-}\left[\rho\left(x, t^{\prime}\right)\right]}{\rho_{i}\left(x, t^{\prime}\right)} d t^{\prime}\right\} \rho_{i}(x, t)\right]$ must be evaluated for every site on the lattice. This means that it is necessary to keep a history of the concentrations for every point on the lattice. For efficiency, it is also convenient to keep the history of the integral that appears in the exponential.

The "short-memory" principle [27] allows us to simplify matters and speed up the computation. The definition of the fractional derivative in Eq. (8) indicates that times closer to the present contribute more than times further in the past. Using a "cut-off" $t_{\text {cut }}$ in how far back one goes in time to calculate the fractional derivative ensures that the numerical evaluation does not slow to halt as $t$ becomes large. One must be careful, however, to choose a value of $t_{\text {cut }}$ which does not interfere appreciably with the results. Further details of the method are discussed in Appendix D.

The numerical integration is carried out on a lattice with 101 nodes and with spacing $\Delta x=1$, which is much smaller than the wavelength of the patterns produced in the examples. The time step used in the Euler-forward integration is $\Delta t=10^{-4}$. Further model parameters are given in the figure captions.

\section{Numerical comparison of subdiffusive and corresponding normally diffusive systems}

\section{Stationary patterns}

As discussed in Sec. III A, for fixed model parameters we expect the same stationary patterns to result from the reaction-subdiffusion system and the corresponding effective Markovian system in the long term. The patterns in the Lengyel-Epstein model shown in the left-hand panel of Fig. 1 confirm this; the amplitude, periodicities, and general shapes of the patterns in the original and the effective systems match. The similarity of the patterns is further evidenced in the Fourier spectra shown in the right-hand panel. We attribute 

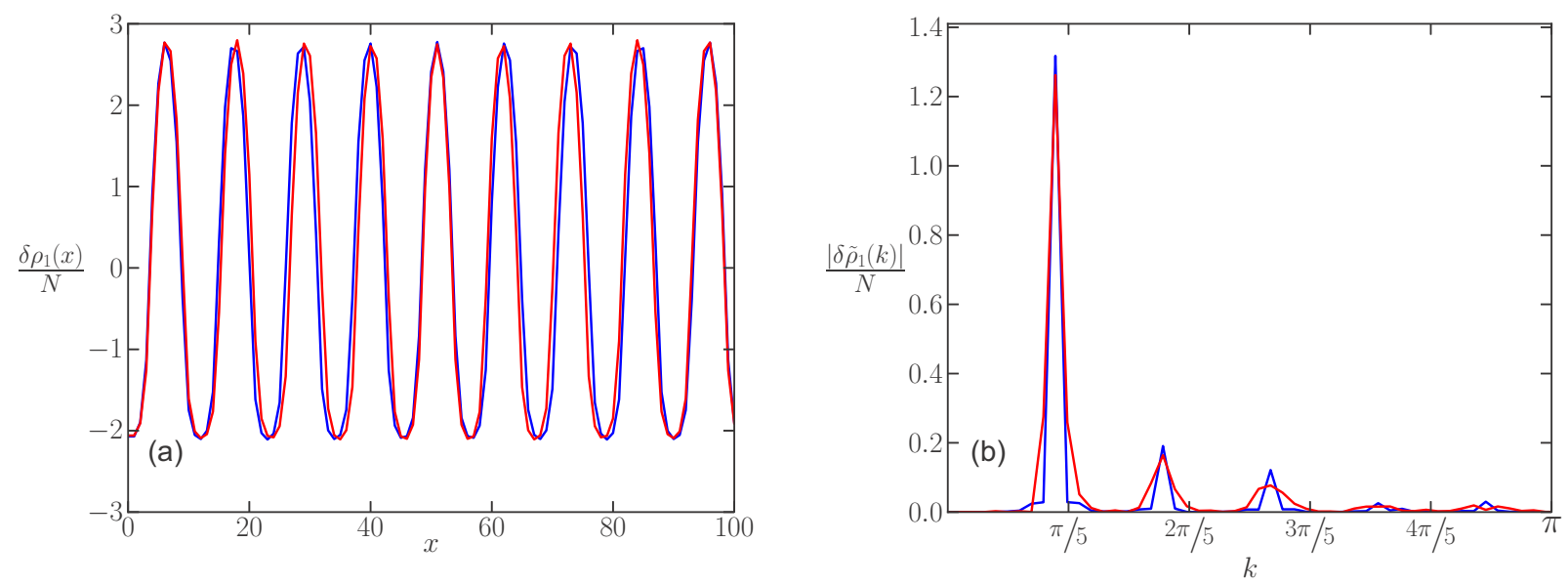

FIG. 1. (a) Comparison of patterns obtained from numerically integrating the full reaction-subdiffusion equation (10), shown as a blue line, and the effective Markovian system in Eq. (16), shown as a red line. Data show deviations from the homogeneous fixed point for species A of the the Lengyel-Epstein model (cf. Sec. IV A). (b) Fourier spectra of these patterns. The model parameters are $a=2, b=0.13, c=1, d=1$, $N=1000, \alpha_{A}=0.5, \alpha_{B}=1, \frac{\sigma_{A}^{2}}{\eta_{A}}=1.05, \frac{\sigma_{B}^{2}}{\eta_{B}}=0.33, p_{A}=0.65, p_{B}=0.294$. Both systems were run until $t=300$ so that the patterns have sufficient time to form. The cut-off time used was $t_{\text {cut }}=10$.

the remaining differences to inaccuracies in the numerical methods. Figure 2 shows that the transient approach to the patterned stationary state in the subdiffusive system differs from that in the effective cross-diffusive system.

\section{Time evolution for large removal rates}

We also deduced in Sec. III C that the original subdiffusive system and the corresponding effective Markovian system have approximately the same dynamics when the particle removal rates are large. In Figs. 3 and 4, we verify this for the case in which one species subdiffuses and the other diffuses normally. If one component diffuses normally $\left(\alpha_{i}=1\right)$, then the evolution equation for this component is identical in both systems. So we only require the subdiffusing component to have a large removal rate in order for the dynamics to be the same in both systems.

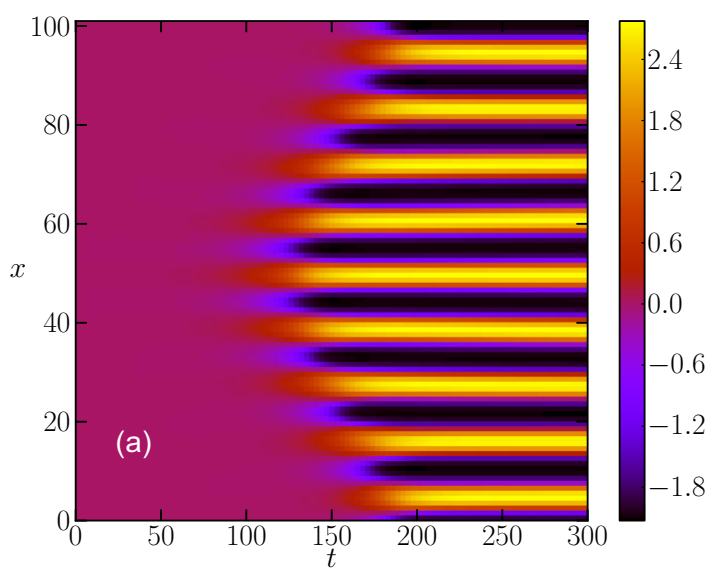

Figure 3 demonstrates the accuracy of the approximation for large removal rates. We compare the transient time evolution of the concentration for the original system and for the effective Markovian system. This is done for two different locations in the spatial domain. Figure 4 shows the agreement between the systems over longer timescales as patterns begin to emerge.

\section{SUMMARY AND DISCUSSION}

In a Markovian reaction-diffusion system, the quantification of the motility of a particular species is comparatively straightforward since one can easily define the diffusion coefficient, which can be related to the rate of change with time of the mean-squared displacement for an individual particle. The situation in subdiffusive systems is more complicated. In this paper we have defined the effective diffusion coefficient

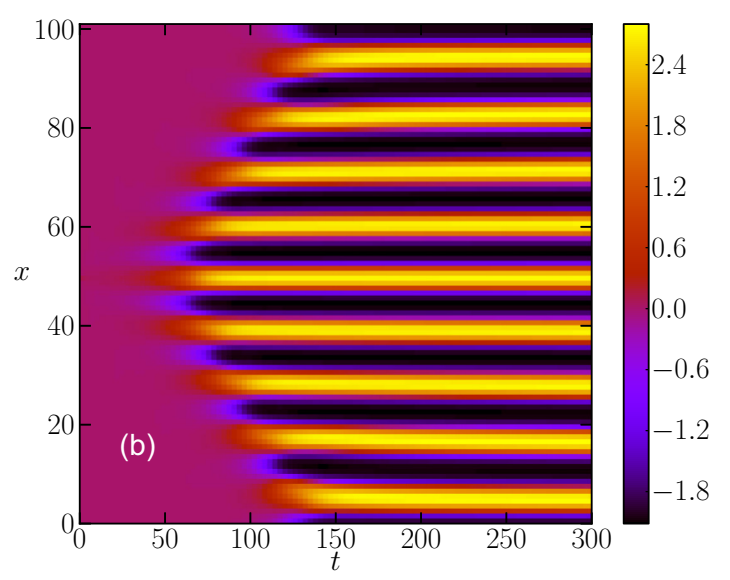

FIG. 2. Comparison of the transient approach to the patterned state in the (a) original and (b) effective Markovian systems for the same model and parameters as in Fig. 1. We show $\delta \rho_{A} / N$ as relief. The patterns are fully formed at roughly $t=150$ in the effective system but take until around $t=200$ to form in the subdiffusive system. The system is initialized at the homogeneous fixed point, with a deviation of magnitude 10 at $x=51$ at $t=0$. The cut-off time used was $t_{\text {cut }}=10$. 

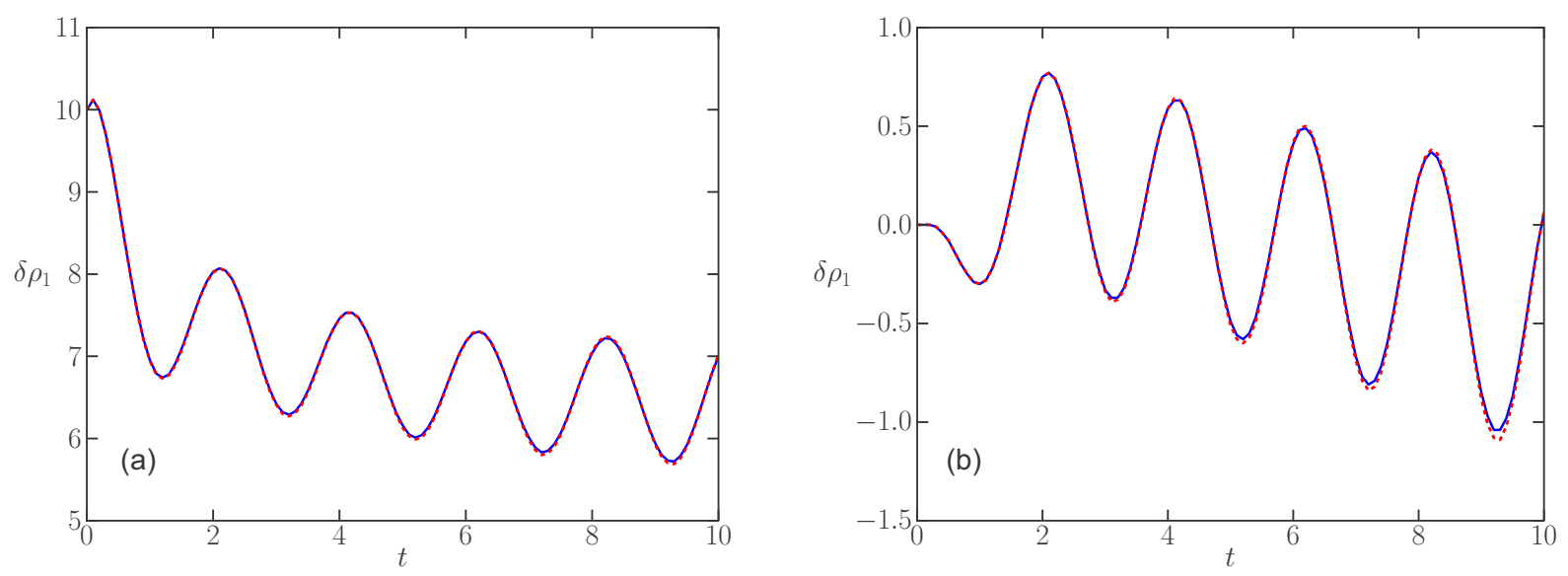

FIG. 3. Comparison of the initial transient behavior in the original and effective Markovian systems for the case of large removal rate for the subdiffusing component. Integration is initialized at the homogeneous fixed point but with a deviation of magnitude 10 at $x=0$ at $t=0$. (a) Time evolution of species A at $x=0$ in the reaction-subdiffusion system (blue line) and the corresponding Markovian system (red dashed line). (b) Time evolution of species A at $x=6$. The model parameters are $a=27, b=4, c=1, d=1, N=1000, \alpha_{A}=0.5, \alpha_{B}=1$, $\frac{\sigma_{A}^{2}}{\eta_{A}}=0.075, \frac{\sigma_{B}^{2}}{\eta_{B}^{\alpha_{B}}}=16.7$. The death rate of the subdiffusing species $\mathrm{A}$ is $p_{A}=20$, the death rate for the normally diffusing species $\mathrm{B}$ is $p_{B}=0.478$. The cut-off time used was $t_{\text {cut }}=1$.

for a reaction-subdiffusion system, and we are now able to compare the motilities of particle species. These effective diffusion coefficients are dependent not only on the anomalous exponents and the typical waiting times of particles but also on the reaction rates. This in turn gives rise to cross-diffusive behavior. Moreover, we have shown that, using the effective diffusion coefficients, the condition for Turing pattern formation in a subdiffusive system can be written in the same form as it would appear in a Markovian system. We have therefore demonstrated that the effective diffusion coefficients are indeed the pertinent quantities with which one can characterize transport effects in a reaction-subdiffusion system.

We emphasize that while the effective Markovian system replicates the behavior in the subdiffusive system in the sta- tionary state, it reproduces the transient dynamical behavior only under special circumstances. Interesting time-dependent phenomena which are peculiar to reaction-subdiffusion systems, such as the failure of front-propagation [38], are not necessarily reproduced fully by the effective system. That being said, we note that the concentration profiles in Ref. [39], which were reported to have unique character due to subdiffusion, can also be produced using an effective Markovian system of the type discussed in this paper. This can be seen through the fact that Eq. (11) in Ref. [39] is a special case of our Eq. (23) for the particular reaction scheme used in that paper.

In Sec. III D we showed that the mean-squared displacement of particles can be thought of as increasing linearly with time even in a subdiffusive system. This view can be taken
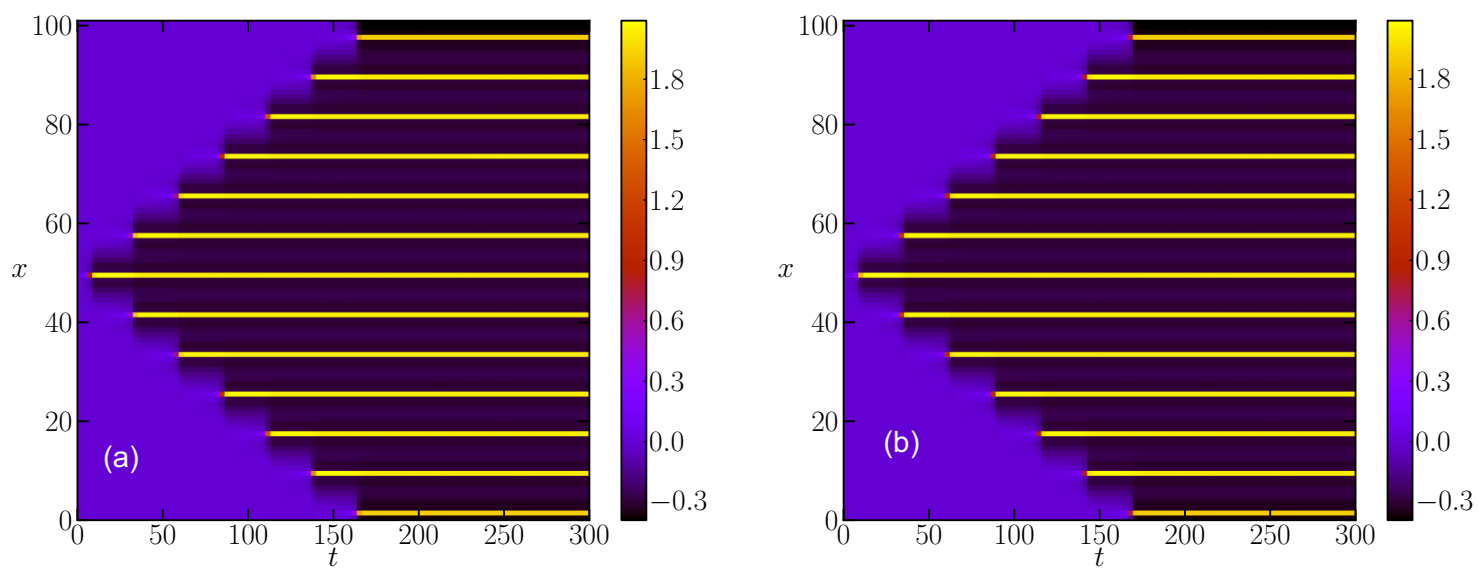

FIG. 4. Comparison of the intermediate-time behavior in the (a) effective Markovian and (b) original systems for the case of large removal rate for the subdiffusing component (see text for details). We show $\delta \rho_{A} / N$ as relief. The system is initialized at the homogeneous fixed point, with a deviation of magnitude 100 at $x=51$ at $t=0$. Model parameters are $a=27, b=4.06, c=1, d=1, N=1000, \alpha_{A}=0.5$, $\alpha_{B}=1, \frac{\sigma_{A}^{2}}{\eta_{A} \alpha_{A}}=0.067, \frac{\sigma_{B}^{2}}{\eta_{B}}=33.3$. The death rate of the subdiffusing species is $p_{A}=20$, the death rate for the normally diffusing species is $p_{B}=0.478$. The cut-off time used was $t_{\text {cut }}=10$. 
if one interprets diffusion as a phenomenon defined by an ensemble of particles, rather than by tracking the motion of individual particles. Different choices for the ensemble can lead to subdiffusive or regular diffusive behavior. In the latter case, the diffusion coefficient, defined from the ensemble view, coincides with the one we obtained from analyzing the Turing instability of the system with anomalous diffusion.

There exist many explanations for the observed crossdiffusive effects in real-life systems. In ecological systems for example, predators pursue their prey and prey avoid their predators [40,41]. Mechanisms leading to cross-diffusive behavior in physical and chemical systems include electrostatic interactions, excluded-volume effects, and complexation [42]. Our analysis suggests another possible mechanism: If a system exhibits memory effects due to anomalous transport, then cross-diffusion can arise when the removal rate of one species of particle depends on the concentration of another species.

A common way of measuring diffusion coefficients in chemical systems experimentally is the Taylor method, which involves monitoring the spread of the concentration of a drop placed in a laminar flow. A Gaussian profile is then fitted to the data in order to estimate the diffusion coefficients $[43,44]$. In such an experiment, individual particles are not tracked and so the diffusive properties are inferred based on macroscopic statistical behavior. Our work would suggest that cross-diffusive coefficients arising in experiments of this kind could possibly originate from, or at least be affected by, non-Markovian transport.
While we restricted most of the discussion to the case of subdiffusion with Mittag-Leffler distributed waiting times, we note that an effective normally diffusive system can be found for general non-Markovian systems described by Eq. (1); the calculations that were carried out starting from Eq. (10) could just as well be performed with Eq. (1). So effective diffusion coefficients can also be found for reaction-diffusion systems with other types of nonstandard diffusion.

\section{ACKNOWLEDGMENTS}

J.W.B. thanks the Engineering and Physical Sciences Research Council (EPSRC) for funding (Grant No. EP/N509565/1). We also thank Francisco Herrarías-Azcué for his helpful comments.

\section{APPENDIX A: CRITERION FOR TURING PATTERNS}

\section{Derivation of the criterion for Turing instability}

In this Appendix we derive the condition in Eq. (15) for the instability of a Fourier mode with wave number $k$. The calculation differs from that in Ref. [22] in that we avoid the use of contour integration; we also consider the case where both species can subdiffuse.

We start from the linearized dynamics in Eq. (14) and proceed using the ansatz

$$
\delta \underline{\tilde{\rho}}(k, t)=e^{\lambda_{k} t} \delta \underline{\tilde{\rho}}(k, 0),
$$

where $\lambda_{k}>0$ is real. Inserting this into Eq. (14) and using Eq. (22), we find

$$
\lambda_{k} \delta \tilde{\rho}_{i}(k, t)=-k^{2} \frac{\sigma_{i}^{2}}{\eta_{i}^{\alpha_{i}}}\left[\left(p_{i}+\lambda_{k}\right)^{1-\alpha_{i}} \delta \tilde{\rho}_{i}(k, t)-\sum_{j} A_{i j} \rho_{i}^{0} \frac{p_{i}^{1-\alpha_{i}}-\left(\lambda_{k}+p_{i}\right)^{1-\alpha_{i}}}{\lambda_{k}} \delta \tilde{\rho}_{j}(k, t)\right]+\sum_{j} f_{i j} \delta \tilde{\rho}_{j}(k, t),
$$

at long times. Equation (A2) can be rewritten in matrix form

$$
\underline{\underline{M_{k}}} \delta \underline{\tilde{\rho}_{k}}=\underline{0} .
$$

In order for the solution to be nontrivial, the matrix $\underline{\underline{M_{k}}}$ must be singular, that is,

$$
\Delta_{k}\left(\lambda_{k}\right) \equiv \operatorname{det} \underline{\underline{M_{k}}}=0 .
$$

Equation (A4) determines the possible values that $\lambda_{k}$ may take. The determinant $\Delta_{k}\left(\lambda_{k}\right)$ can be written as

$$
\begin{aligned}
\Delta_{k}\left(\lambda_{k}\right)= & \left\{\lambda_{k}+k^{2} \frac{\sigma_{1}^{2}}{\eta_{1}^{\alpha_{1}}}\left[\left(\lambda_{k}+p_{1}\right)^{1-\alpha_{1}}+A_{11} \rho_{1}^{0} \frac{\left(\lambda_{k}+p_{1}\right)^{1-\alpha_{1}}-p_{1}^{1-\alpha_{1}}}{\lambda_{k}}\right]-f_{11}\right\} \\
& \times\left\{\lambda_{k}+k^{2} \frac{\sigma_{2}^{2}}{\eta_{2}^{\alpha_{2}}}\left[\left(\lambda_{k}+p_{2}\right)^{1-\alpha_{2}}+A_{22} \rho_{2}^{0} \frac{\left(\lambda_{k}+p_{2}\right)^{1-\alpha_{2}}-p_{2}^{1-\alpha_{2}}}{\lambda_{k}}\right]-f_{22}\right\} \\
& -\left[k^{2} \frac{\sigma_{2}^{2}}{\eta_{2}^{\alpha_{2}}} A_{21} \rho_{2}^{0} \frac{\left(\lambda_{k}+p_{2}\right)^{1-\alpha_{2}}-p_{2}^{1-\alpha_{2}}}{\lambda_{k}}-f_{21}\right]\left[k^{2} \frac{\sigma_{1}^{2}}{\eta_{1}^{\alpha_{1}}} A_{12} \rho_{1}^{0} \frac{\left(\lambda_{k}+p_{1}\right)^{1-\alpha_{1}}-p_{1}^{1-\alpha_{1}}}{\lambda_{k}}-f_{12}\right] .
\end{aligned}
$$

We note that this expression does not reduce exactly to the one given in Ref. [22], see Appendix A 2. The mode with wave number $k$ is guaranteed to be unstable if Eq. (A4) has a real and positive root.

For $0<\alpha_{i}<1$, we have $\Delta_{k}\left(\lambda_{k}\right) \approx \lambda_{k}^{2}$ for large real $\lambda_{k}$, i.e., $\Delta_{k}\left(\lambda_{k}\right)$ is positive. Additionally, the function $\Delta_{k}\left(\lambda_{k}\right)$ is continuous. Hence, if $\Delta_{k}(0)<0$, then there must be at least one real positive zero. In other words, $\Delta_{k}(0)<0$ is a sufficient condition for instability. This leads to Eq. (15). We refer to Ref. [22] for arguments as to why this is not only a sufficient condition but also necessary. 

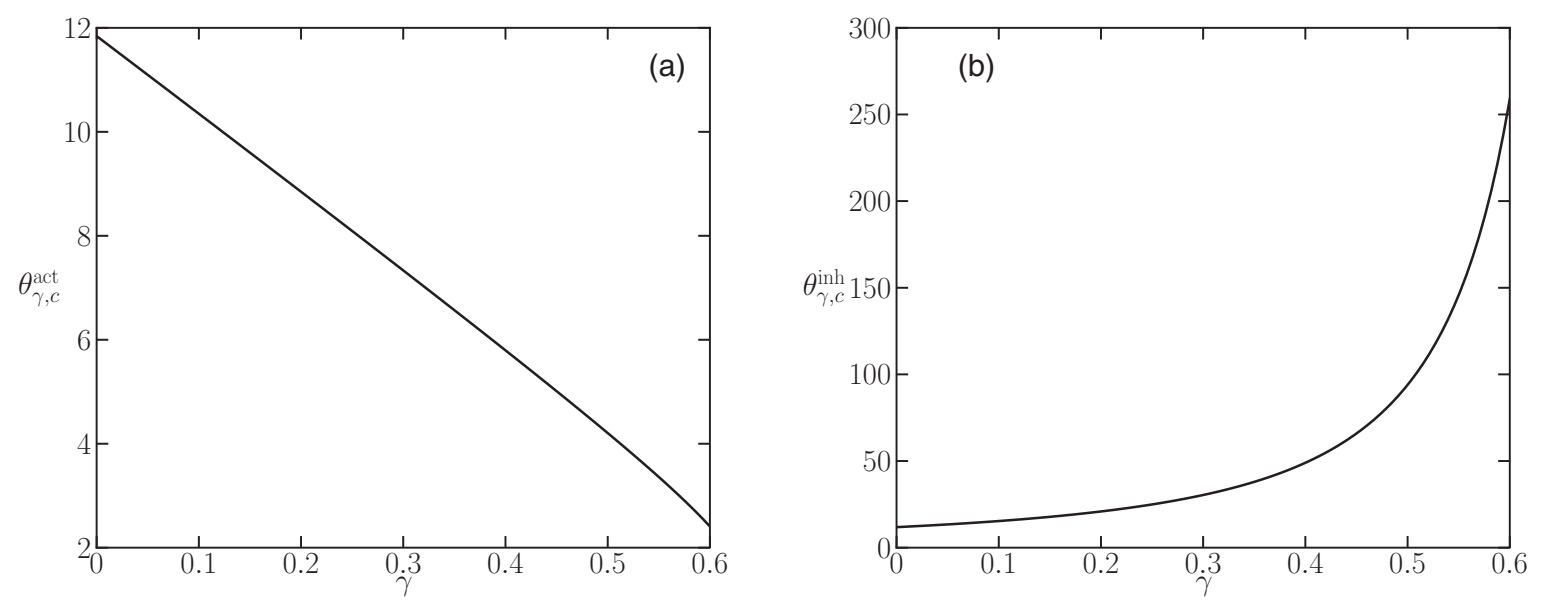

FIG. 5. Value of $\theta_{\gamma}$ marking the Turing instability in the Lengyel-Epstein model as a function of $\gamma=1-\alpha$. Model parameters $a^{\prime}=50$ and $b^{\prime}=40$. Panels (a) and (b) correspond to Figs. 2 and 3 in Ref. [20], respectively.

\section{Correction to the Turing instability criterion in Ref. [22] and verification in simulations}

In this Appendix, we point out a small (but consequential) error which was made in the calculation of the Turing instability in Ref. [22] and built on in Ref. [20]. We also discuss some of the most important consequences.

In going from Eq. (29) to (31) in Ref. [22], a sign error was made in one of the terms. Eq. (31) in Ref. [22] should read

$$
\begin{aligned}
u \delta \rho_{1}(k, u)= & \delta \rho_{1}(k, t=0)+\frac{\sigma_{1}^{2} k^{2} \rho_{1}^{0} p^{1-\alpha} h_{\alpha}(u, p)}{u \eta_{1}^{\alpha}}\left[A_{1} \delta \rho_{1}(k, u)+A_{2} \rho_{2}(k, u)\right]-\frac{\sigma_{1}^{2} k^{2}(u+p)^{1-\alpha}}{\eta_{1}^{\alpha}} \\
& +R_{11}^{+}\left(\rho^{0}\right) \delta \rho_{1}(x, t)+R_{12}^{+}\left(\rho^{0}\right) \delta \rho_{2}(x, t)-R_{11}^{-}\left(\rho^{0}\right) \delta \rho_{1}(x, t)-R_{12}^{-}\left(\rho^{0}\right) \delta \rho_{2}(x, t) .
\end{aligned}
$$

It is the terms involving $A_{1}$ and $A_{2}$ in Eq. (A6) which have the incorrect sign in Ref. [22].

In Ref. [20], conditions for Turing pattern formation are given in Eqs. (24) and (31). These are a special case of our more general Eq. (25), in that only one subdiffusing species is considered. Given the sign error in Ref. [22], Eqs. (24) and (31) in Ref. [20] attract corrections as well.

Following Ref. [22], we denote species 1 as the subdiffusing species with per capita removal rate $p$ and total reaction rate $f$ and species 2 as the normally diffusing species with total reaction rate $g$. We define $\gamma=1-\alpha$ and $\theta_{\gamma}=\frac{\eta_{1}^{\alpha} \sigma_{2}^{2}}{\eta_{2} \sigma_{1}^{2} p^{1-\alpha}}=\frac{\hat{D}_{2}\left(\rho^{0}\right)}{\hat{D}_{1}\left(\rho^{0}\right)}$, the ratio of the effective diffusion coefficients $\hat{D}$ evaluated in the homogeneous steady state. The condition for Turing instability can be written $\theta_{\gamma}>\theta_{\gamma, c}$, where the critical value $\theta_{\gamma, c}$ can take two forms, depending on whether the activator or the inhibitor is subdiffusing.

For a subdiffusing activator one has

$$
\begin{aligned}
\theta_{\gamma, c}^{\mathrm{act}}= & \frac{1}{f_{1}^{2} p^{2}}\left\{-2 f_{2} g_{1} p^{2}+f_{1} g_{2} p^{2}+A_{2} f_{1} g_{1} p \rho_{1}^{0} \gamma-2 A_{1} f_{2} g_{1} p \rho_{1}^{0} \gamma+A_{1} f_{1} g_{2} p \rho_{1}^{0} \gamma\right. \\
& \left.+2 \sqrt{g_{1}\left(f_{2} g_{1}-f_{1} g_{2}\right) p^{2}\left(p+A_{1} \rho_{1}^{0} \gamma\right)\left[f_{2}\left(p+A_{1} \rho_{1}^{0} \gamma\right)-A_{2} f_{1} \rho_{1}^{0} \gamma\right]}\right\}
\end{aligned}
$$

where as for the case of a subdiffusing inhibitor the condition reads

$$
\begin{aligned}
\theta_{\gamma, c}^{\text {inh }}= & \left(\frac { 1 } { f _ { 1 } ^ { 2 } p ^ { 2 } } \left\{-2 f_{2} g_{1} p^{2}+f_{1} g_{2} p^{2}+A_{2} f_{1} g_{1} p \rho_{1}^{0} \gamma-2 A_{1} f_{2} g_{1} p \rho_{1}^{0} \gamma+A_{1} f_{1} g_{2} p \rho_{1}^{0} \gamma\right.\right. \\
& \left.\left.-2 \sqrt{g_{1}\left(f_{2} g_{1}-f_{1} g_{2}\right) p^{2}\left(p+A_{1} \rho_{1}^{0} \gamma\right)\left[f_{2}\left(p+A_{1} \rho_{1}^{0} \gamma\right)-A_{2} f_{1} \rho_{1}^{0} \gamma\right]}\right\}\right)^{-1} .
\end{aligned}
$$

These expressions are different from the ones in Ref. [20] in that the signs of $A_{1}$ and $A_{2}$ have both been inverted.

The sign error has important consequences to the conclusions drawn in Ref. [20]. In fact, with the error taken into account the phase diagrams shown in Figs. 2 and 3 in Ref. [20] change considerably. We present the corrected phase diagrams in Fig. 5.

Verification in simulations. To the best of our knowledge the theoretical predictions in Ref. [20] have not been tested numerically. To verify Eq. (A8) we use the method described in the main text and in Appendix D. The version of the Lengyel-Epstein model used in Ref. [20] can be obtained from the setup described in Eqs. (31) and (32) by the rescaling $\rho_{A}^{\prime}=\frac{\rho_{A}}{N}, \rho_{B}^{\prime}=\frac{c \rho_{2}}{N}$ and $d=b=1, c=b^{\prime}, a=a^{\prime}$ (dashed quantities are the ones used in Ref. [20]). 

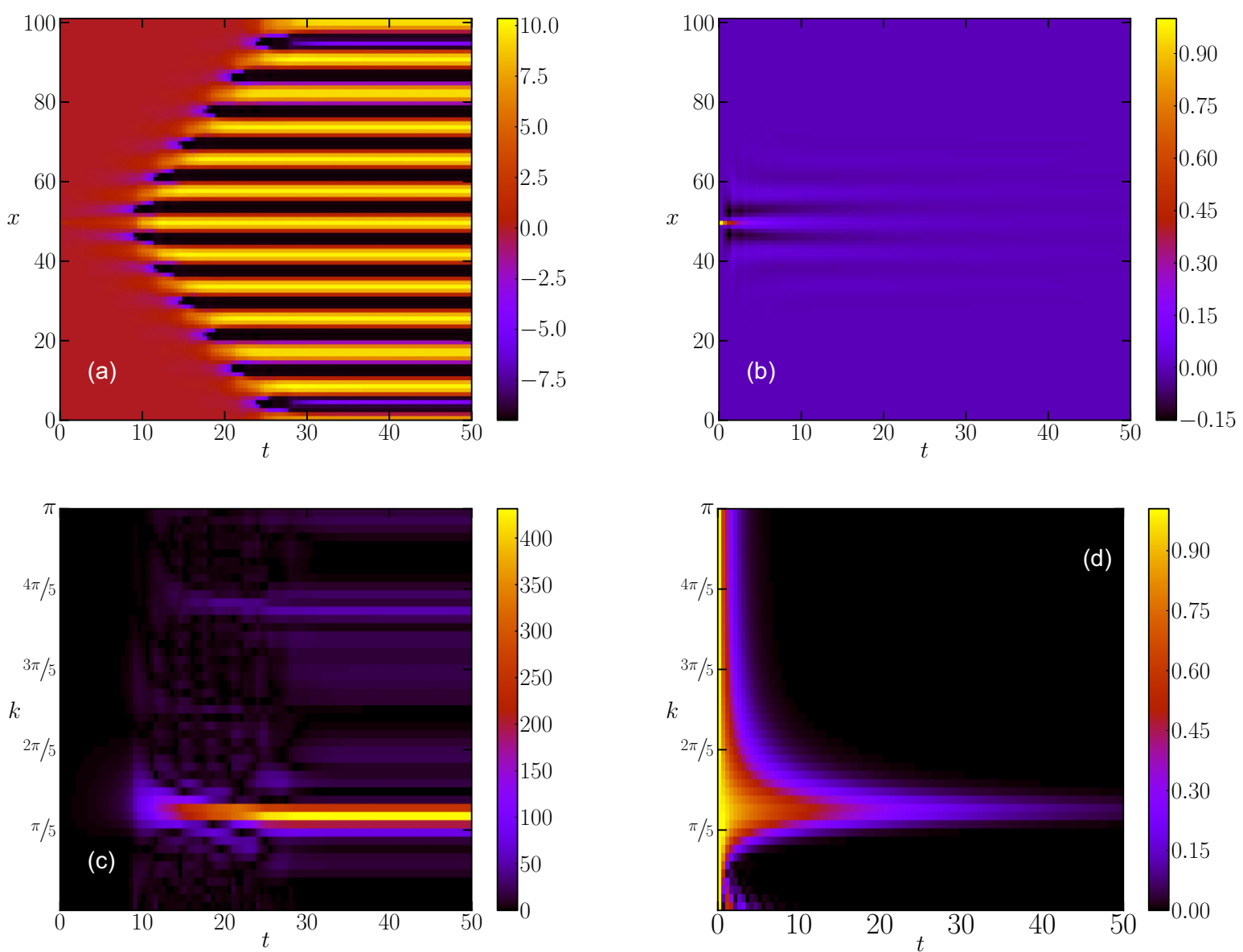

FIG. 6. Results from numerical integration of the Lengyel-Epstein model with subdiffusing activator, subject to an initial small perturbation around the homogeneous fixed point at $x=51$. The upper panels show deviations from the fixed point in real space, the lower panels are in Fourier space. [(a) and (c)] Model parameters are above the threshold for Turing pattern formation in Fig. 5, but below the threshold predicted in Fig. 2 of Ref. [20] $\left(a^{\prime}=50, b^{\prime}=40, \gamma=0.5, \eta_{1}=0.02, \eta_{2}=0.01\right.$, resulting in $\left.\theta_{\gamma}=\eta_{1}^{\alpha} / \eta_{2} p^{1-\alpha}=6.32\right)$. Pattern formation is clearly visible in real space and in Fourier space. [(b) and (d)] Model parameters $a^{\prime}, b^{\prime}$, and $\gamma$ are as on the left, but $\eta_{1}=0.008, \eta_{2}=0.01$, resulting in $\theta_{\gamma}=4$. This is just below the threshold for pattern formation in Fig. 5. The perturbation decays, and no patterns are found.

This yields

$$
f^{\prime}\left(\rho^{\prime}\right)=a^{\prime}-\rho_{A}^{\prime}-4 \frac{\rho_{A}^{\prime} \rho_{B}^{\prime}}{1+\left(\rho_{A}^{\prime}\right)^{2}}, \quad g^{\prime}\left(\rho^{\prime}\right)=b^{\prime}\left[\rho_{A}^{\prime}-\frac{\rho_{A}^{\prime} \rho_{B}^{\prime}}{1+\left(\rho_{A}^{\prime}\right)^{2}}\right], \quad \frac{R_{A}^{\prime}}{\rho_{A}^{\prime}}=1+4 \frac{\rho_{B}^{\prime}}{1+\left(\rho_{A}^{\prime}\right)^{2}} .
$$

An example of the outcome of the numerical integration is shown in Fig. 6. One finds patterned stationary states only when $\theta_{\gamma}$ is greater than the critical value given in Eq. (A8). At the same time patterns can be observed below the threshold given in Fig. 2 of Ref. [20], confirming our version of the calculation of the phase diagram.

Importantly, these numerical results demonstrate that, in this model, one can observe Turing patterns for lower values of $\theta_{\gamma}$ when the activator is subdiffusing than when both reactants are diffusing normally or when the inhibitor subdiffuses. The opposite is reported in Ref. [20].

\section{APPENDIX B: LIMIT OF LARGE REMOVAL RATES}

In this Appendix, we derive Eq. (27). First, we rewrite Eq. (8) in the following form:

$$
{ }_{0} D_{t}^{1-\alpha} f(t)=\frac{1}{\Gamma(\alpha)} \frac{\partial}{\partial t} \int_{0}^{t} \frac{f(t-\tau)}{\tau^{1-\alpha}} d \tau=\frac{1}{\Gamma(\alpha)}\left[t^{\alpha-1} f(0)+\int_{0}^{t} \frac{\frac{\partial}{\partial t} f(t-\tau)}{\tau^{1-\alpha}} d \tau\right] .
$$

Thus we have

$$
{ }_{0} D_{t}^{1-\alpha_{i}}\left\{\rho_{i}(x, t) e^{\int_{0}^{t} p_{i}\left(x, t^{\prime}\right) d t^{\prime}}\right\}=\frac{1}{\Gamma(\alpha)}\left\{t^{\alpha-1} \rho_{i}(x, 0)+\int_{0}^{t} \tau^{\alpha-1}\left[\frac{\partial \rho_{i}(x, t-\tau)}{\partial t}+\rho_{i}(x, t-\tau) p_{i}(x, t-\tau)\right] e^{\int_{0}^{t-\tau} p_{i}\left(x, t^{\prime}\right) d t^{\prime}} d \tau\right\} .
$$


We now assume that the removal rate for particles of species $i$ at the homogeneous fixed point is large. So we would like to find a series expansion for integrals of the form

$$
\int_{0}^{t} \tau^{\alpha-1} f(t-\tau) e^{M g(t-\tau)} d \tau
$$

where $M$ is a large dimensionless parameter. This can be done using a method analogous to Laplace's method [45].

We presume that the functions $f$ and $g$ can be expanded as Taylor series, which converge for $0<\tau<t$, such that

$$
f(t-\tau)=f(t)-\tau f^{\prime}(t)+\frac{1}{2 !} \tau^{2} f^{\prime \prime}(t)+\cdots, \quad g(t-\tau)=g(t)-\tau g^{\prime}(t)+\frac{1}{2 !} \tau^{2} g^{\prime \prime}(t)+\cdots .
$$

In our case, $g^{\prime}(t)>0$. Noting that the series for the exponential function is absolutely convergent for any value, one then obtains

$$
\begin{aligned}
\int_{0}^{t} \tau^{\alpha-1} f(t-\tau) e^{M g(t-\tau)} d \tau= & \int_{0}^{t} \tau^{\alpha-1}\left[f(t)-\tau f^{\prime}(t)+\frac{1}{2 !} \tau^{2} f^{\prime \prime}(t)+\cdots\right] \\
& \times\left\{1+M\left[\frac{1}{2 !} \tau^{2} g^{\prime \prime}(t)-\frac{1}{3 !} \tau^{3} g^{\prime \prime \prime}(t)+\cdots\right]+\frac{1}{2 !} M^{2}\left[\frac{1}{2 !} \tau^{2} g^{\prime \prime}(t)-\frac{1}{3 !} \tau^{3} g^{\prime \prime \prime}(t)+\cdots\right]+\cdots\right\} \\
& \times e^{M g(t)} e^{-M g^{\prime}(t) \tau} d \tau .
\end{aligned}
$$

We now note the following:

$$
\int_{0}^{t} \tau^{\alpha+n-1} e^{-M \tau g^{\prime}(t)} d \tau=\left[g^{\prime}(t) M\right]^{-(\alpha+n)} \gamma\left(\alpha+n, g^{\prime}(t) M t\right)
$$

where $\gamma(s, x)=\int_{0}^{x} t^{s-1} e^{-t} d t$ is the lower incomplete gamma function. Expanding further in Equation (B5) and reorganizing terms, one obtains the following asymptotic series:

$$
\begin{aligned}
& \int_{0}^{t} \tau^{\alpha-1} f(t-\tau) e^{M g(t-\tau)} d \tau \\
& \quad=e^{M g(t)}\left[M g^{\prime}(t)\right]^{-\alpha}\left\{f(t) \gamma\left(\alpha, g^{\prime}(t) M t\right)+\frac{\left[\frac{f(t) g^{\prime \prime}(t)}{2 ! g^{\prime}(t)^{2}} \gamma\left(\alpha+2, g^{\prime}(t) M t\right)-\frac{f^{\prime}(t)}{g^{\prime}(t)} \gamma\left(\alpha+1, g^{\prime}(t) M t\right)\right]}{M}+\cdots\right\}
\end{aligned}
$$

The lower incomplete gamma function fulfills following relation [30]:

$$
\gamma(s, x)=\Gamma(s)-\Gamma(s, x)=\Gamma(s)-e^{-x} x^{s-1}-e^{-x} x^{s-1} \sum_{k=1}^{\infty} \frac{\prod_{l=1}^{k}(s-l)}{x^{k}} .
$$

Although the arguments of the lower incomplete gamma functions in Eq. (B7) depend on $M$, one can see from Eq. (B8) that the series expansion for the lower incomplete gamma function consists of a constant term plus a correction term proportional to $e^{-g^{\prime}(t) M t}$. That is, $\gamma\left(\alpha+n, g^{\prime}(t) M t\right)=\Gamma(\alpha+n)+O\left\{e^{-g^{\prime}(t) M t}\left[g^{\prime}(t) M t\right]^{\alpha+n-1}\right\}$. This correction always decays more quickly than $M^{\alpha+r}$ for any $r$. Therefore, we can write

$$
\int_{0}^{t} \tau^{\alpha-1} f(t-\tau) e^{M g(t-\tau)} d \tau=e^{M g(t)}\left[M g^{\prime}(t)\right]^{-\alpha}\left[f(t) \Gamma(\alpha)+O\left(\frac{1}{M}\right)\right] .
$$

For our problem, we presume that $p_{i}(x, t)=M s_{i}(x, t)$, where $M \gg 1$ represents the limit of large removal rates and where $s_{i}(x, t)=O\left(M^{0}\right)$. We obtain

$$
\begin{aligned}
{ }_{0} D_{t}^{1-\alpha_{i}}\left[\rho_{i}(x, t) e^{\int_{0}^{t} p_{i}\left(x, t^{\prime}\right) d t^{\prime}}\right] & =\frac{1}{\Gamma(\alpha)}\left\{t^{\alpha-1} \rho_{i}(x, 0)+\int_{0}^{t} \tau^{\alpha-1}\left[\frac{\partial \rho_{i}(x, t-\tau)}{\partial t}+\rho_{i}(x, t-\tau) p_{i}(x, t-\tau)\right] e^{\int_{0}^{t-\tau} p_{i}\left(x, t^{\prime}\right) d t^{\prime}} d \tau\right\} \\
& =e^{\int_{0}^{t} p_{i}\left(x, t^{\prime}\right) d t^{\prime}} p_{i}(x, t)^{1-\alpha} \rho_{i}(x, t)\left[1+O\left(\frac{1}{M}\right)\right]
\end{aligned}
$$

since $\frac{\partial \rho_{i}(x, t)}{\partial t}=O\left(M^{0}\right)$ and the term $\frac{1}{\Gamma(\alpha)} t^{\alpha-1} \rho_{i}(x, 0)$ is exponentially smaller than the rest. Hence, for large $M$ [or large $p_{i}(x, t)$, we arrive at the approximation in Eq. (27). 


\section{APPENDIX C: MEAN-SQUARED DISPLACEMENTS}

In this Appendix, we derive Eqs. (29) and (30). We start from Eq. (28) and take combined Fourier and Laplace transforms with respect to position and time, respectively. We find

$$
\hat{\tilde{C}}_{i}(k, u)=\frac{1}{u+\frac{\sigma_{i}^{2}}{\eta_{i}^{\alpha}} k^{2}\left[u+(1-\theta) p_{i}\right]^{1-\alpha_{i}}} .
$$

Noting that $P_{i}(x, t)=C_{i}(x, t)$ for $\theta=0$, one has $\left\langle x^{2}\right\rangle=\int C_{i}(x, t) x^{2} d x$ for both $\theta=0$ and $\theta=1$. Using $\left\langle x^{2}\right\rangle=$ $-\left.\frac{\partial^{2}}{\partial k^{2}} \tilde{C}_{i}(k, t)\right|_{k=0}$, one then obtains

$$
\mathcal{L}_{t}\left\{\left\langle x^{2}(t)\right\rangle\right\}(u)=2 \frac{\sigma_{i}^{2}}{\eta_{i}^{\alpha}} \frac{\left[u+(1-\theta) p_{i}\right]^{1-\alpha_{i}}}{u^{2}} .
$$

Inverting the Laplace transform one finds for $\theta=0$

$$
\left\langle x^{2}(t)\right\rangle=2 \frac{\sigma_{i}^{2}}{\eta_{i}^{\alpha}} e^{-p_{i} t}{ }_{0} D_{t}^{1-\alpha_{i}}\left\{e^{p_{i} t} t\right\}=2 \frac{\sigma_{i}^{2}}{\eta_{i}^{\alpha}} \frac{1}{\Gamma\left(\alpha_{i}\right)} p_{i}^{-\alpha_{i}}\left[\left(p_{i} t+1\right) \gamma\left(\alpha_{i}, p_{i} t\right)-\gamma\left(\alpha_{i}-1, p_{i} t\right)\right] \approx 2 \frac{\sigma_{i}^{2}}{\eta_{i}^{\alpha}} p_{i}^{1-\alpha_{i}} t,
$$

where we have used an approximation similar to Eq. (22) in the limit $p_{i} t \gg 1$.

For $\theta=1$, on the other hand, one finds, using Eq. (C2) and the fact that $\mathcal{L}_{t}(u)\left\{t^{\alpha}\right\}=\Gamma(1+\alpha) u^{-(1+\alpha)}$,

$$
\left\langle x^{2}(t)\right\rangle=2 \frac{\sigma_{i}^{2}}{\eta_{i}^{\alpha}} \frac{1}{\Gamma\left(1+\alpha_{i}\right)} t^{\alpha_{i}}
$$

So one obtains a subdiffusive law for the mean-squared displacement when only the surviving particles are included in the ensemble.

\section{APPENDIX D: FURTHER DETAILS ON THE NUMERICAL INTEGRATION OF THE REACTION-SUBDIFFUSION EQUATION}

The algorithm for the numerical integration of Eq. (10) operates on a lattice with spacing $\Delta x$ and in discretized time with time step $\Delta t$. It can be stated as follows:

(i) Initialize arrays which can store the histories of particle concentrations for each lattice site as well as the history of the integral $I(x, t)=\int_{0}^{t} \frac{R_{i}^{-}\left[\rho\left(x, t^{\prime}\right)\right]}{\rho_{i}\left(x, t^{\prime}\right)} d t^{\prime}$. Choose a starting configuration for the system. Initialize $I(x, 0)=0$. Choose the number of steps after which to disregard the contribution to the integration: $j_{\text {cut }}$.

(ii) For each site $x$, increment the value of the integral $I(x, t)$ according to

$$
I(x, j \Delta t)=I[x,(j-1) \Delta t]+\Delta t \frac{R_{i}^{-}[\rho(x,(j-1) \Delta t)]}{\rho_{i}[x,(j-1) \Delta t]},
$$

and keep a record of all previous values of this quantity, up to the cut-off.

(iii) For each site $x$, calculate the quantity

$$
\begin{aligned}
F(x, j \Delta t) & =\exp (-I(x, j \Delta t))_{0} D_{j \Delta t}^{1-\alpha}\left\{\exp [I(x, j \Delta t)] \rho_{i}(x, j \Delta t)\right\} \\
& \approx \frac{1}{(\Delta t)^{\alpha}} \sum_{n=\max \left(j-j_{\text {cut }}, 0\right)}^{j}(-1)^{n}\left(\begin{array}{l}
\alpha \\
n
\end{array}\right) \exp [-I(x, n \Delta t)] \rho_{i}[x,(j-n) \Delta t],
\end{aligned}
$$

which uses the Grünwald-Letnikov derivative in Eq. (33) and the histories of the quantities $I(x, t), \rho_{i}(x, t)$.

(iv) Increment the concentrations according to

$$
\begin{aligned}
\rho_{i}(x, j \Delta t) & =\rho_{i}[x,(j-1) \Delta t]+\Delta t \frac{\sigma_{i}^{2}}{\eta_{i}^{\alpha_{i}}}\{F[x+\Delta x,(j-1) \Delta t]+F[x-\Delta x,(j-1) \Delta t]-2 F[x,(j-1) \Delta t]\} \\
& +\Delta t R_{i}^{+}[\rho(x,(j-1) \Delta t)]-\Delta t R_{i}^{-}[\rho(x,(j-1) \Delta t)],
\end{aligned}
$$

and keep a record of all previous values of these quantities, up to the cut-off.

(v) Go to (ii).

With regards to choosing the cut-off time $t_{\text {cut }}$, a simple test as to whether the cut-off is suitably long is to evaluate $e^{-p_{i} t}{ }_{0} D_{t}^{1-\alpha_{i}}\left[e^{p_{i} t}\right]$ and ensure that this agrees with the expected analytical result of $p_{i}^{1-\alpha_{i}}$ for long times. Using an infinite $t_{\text {cut }}$ would clearly be the most accurate choice of cut-off. That said, we found that it was always possible to find a finite cut-off time, which greatly increased the efficiency of the calculations and did not interfere with the results. We tested in selected examples that identical results are obtained for all intents and purposes if no cut-off is used. 
Additionally, we note that more sophisticated methods of evaluating the Grünwald-Letnikov derivative do exist [46-48], but we found that the above method was sufficient for our purposes.

[1] A. M. Turing, Phil. Trans. 237, 37 (1952).

[2] M. C. Cross and P. C. Hohenberg, Rev. Mod. Phys. 65, 851 (1993).

[3] N. F. Britton, Reaction-Diffusion Equations and Their Applications to Biology (Academic Press, London, 1986).

[4] S. Kondo and T. Miura, Science 329, 1616 (2010).

[5] J. D. Murray, Mathematical Biology II: Spatial Models and Biomedical Applications (Springer-Verlag, New York, 2001).

[6] M. Von Smoluchowski, Ann. Phys. 326, 756 (1906).

[7] A. Einstein, Ann. Phys. 17, 549 (1905).

[8] R. Metzler and J. Klafter, Phys. Rep. 339, 1 (2000).

[9] V. Mendez, S. Fedotov, and W. Horsthemke, ReactionTransport Systems: Mesoscopic Foundations, Fronts, and Spatial Instabilities (Springer-Verlag, New York, 2010).

[10] R. Klages, G. Radons, and I. M. Sokolov, Anomalous Transport: Foundations and Applications (Wiley-VCH, Weinheim, 2008).

[11] M. O. Vlad and J. Ross, Phys. Rev. E 66, 061908 (2002).

[12] J. Fort, D. Jana, and J. Humet, Phys. Rev. E 70, 031913 (2004).

[13] K. Seki, M. Wojcik, and M. Tachiya, J. Chem. Phys. 124, 044702 (2006).

[14] S. B. Yuste, E. Abad, and K. Lindenberg, Phys. Rev. E 82, 061123 (2010).

[15] S. Fedotov and S. Falconer, Phys. Rev. E 89, 012107 (2014).

[16] M. Cross and H. Greenside, Pattern Formation and Dynamics in Nonequilibrium Systems (Cambridge University Press, Cambridge, UK, 2009).

[17] P. S. Burada, P. Hänggi, F. Marchesoni, G. Schmid, and P. Talkner, Chem. Phys. Chem. 10, 45 (2009).

[18] P. Reimann, C. Van den Broeck, H. Linke, P. Hänggi, J. M. Rubi, and A. Pérez-Madrid, Phys. Rev. Lett. 87, 010602 (2001).

[19] P. Reimann, C. Van den Broeck, H. Linke, P. Hänggi, J. M. Rubi, and A. Pérez-Madrid, Phys. Rev. E 65, 031104 (2002).

[20] A. Yadav, S. M. Milu, and W. Horsthemke, Phys. Rev. E 78, 026116 (2008).

[21] F. Sagués, V. P. Shkilev, and I. M. Sokolov, Phys. Rev. E 77, 032102 (2008).

[22] A. Yadav and W. Horsthemke, Phys. Rev. E 74, 066118 (2006).

[23] F. Mainardi and R. Goreflo, J. Comput. Appl. Math. 118, 283 (2000).

[24] R. Gorenflo, A. A. Kilbas, F. Mainardi, S. V. Rogosin et al., Mittag-Leffler Functions, Related Topics and Applications (Springer-Verlag, New York, 2014), Vol. 2.

[25] M. F. Shlesinger, Eur. Phys. J. B 90, 93 (2017).

[26] S. Fedotov and S. Falconer, Phys. Rev. E 85, 031132 (2012).
[27] I. Podlubny, Fractional Differential equations: An Introduction to Fractional Derivatives, Fractional differential Equations, to Methods of Their Solution and Some of Their Applications (Elsevier, London, 1998), Vol. 198.

[28] A. Madzvamuse, H. S. Ndakwo, and R. Barreira, J. Math. Biol. 70, 709 (2015).

[29] G. Gambino, M. Lombardo, and M. Sammartino, Nonlinear Anal. Real World Appl. 14, 1755 (2013).

[30] W. Magnus, F. Oberhettinger, and R. P. Soni, Formulas and Theorems for the Special Functions of Mathematical Physics (Springer-Verlag, New York, 2013), Vol. 52.

[31] I. Lengyel, G. Rabai, and I. R. Epstein, J. Am. Chem. Soc. 112, 9104 (1990).

[32] V. Castets, E. Dulos, J. Boissonade, and P. De Kepper, Phys. Rev. Lett. 64, 2953 (1990).

[33] I. Lengyel and I. R. Epstein, Science 251, 650 (1991).

[34] P. Zhuang, F. Liu, V. Anh, and I. Turner, IMA J. Appl. Math. 74, 645 (2009).

[35] M. Dehghan, M. Abbaszadeh, and A. Mohebbi, J. Comp. Appl. Math. 280, 14 (2015).

[36] B. Yu, X. Jiang, and H. Xu, Numer. Algorithms 68, 923 (2015).

[37] R. Scherer, S. L. Kalla, Y. Tang, and J. Huang, Comput. Math. Appl. 62, 902 (2011).

[38] D. Froemberg, H. Schmidt-Martens, I. M. Sokolov, and F. Sagués, Phys. Rev. E 78, 011128 (2008).

[39] D. Froemberg and I. M. Sokolov, Phys. Rev. Lett. 100, 108304 (2008).

[40] B. Dubey, B. Das, and J. Hussain, Ecol. Modell. 141, 67 (2001).

[41] V. N. Biktashev, J. Brindley, A. V. Holden, and M. A. Tsyganov, Chaos 14, 988 (2004).

[42] V. K. Vanag and I. R. Epstein, Phys. Chem. Chem. Phys. 11, 897 (2009).

[43] G. Taylor, Proc. Royal Soc. A 219, 186 (1953).

[44] W. E. Price, J. Chem. Soc., Faraday Trans. 1 84, 2431 (1988).

[45] F. W. J. Olver, Asymptotics and Special Functions (Academic Press, New York, 1997).

[46] C. L. MacDonald, N. Bhattacharya, B. P. Sprouse, and G. A. Silva, J. Comput. Phys. 297, 221 (2015).

[47] F. Zeng, I. Turner, and K. Burrage, J. Sci. Comput. 77, 283 (2018).

[48] C. Li and A. Chen, Int. J. Comput. Math. 95, 1048 (2018).

Correction: The copyright license statement was presented incorrectly because of a publication release error and has been fixed.

Correction: The previously published Fig. 5 contained a plotting error and has been fixed. 